Pacific

Journal of

Mathematics

\title{
THE VECTOR BUNDLE DECOMPOSITION
}

Joshua A. Leslie And QINGQi Yue 


\title{
THE VECTOR BUNDLE DECOMPOSITION
}

\author{
Joshua A. Leslie AND QINGQI Yue
}

In this paper, we studied the real vector bundle decomposition problem. We first give a general decomposition result which relates a given vector bundle to some cohomology classes with local coefficients in the homotopy group of a Grassmann manifold; it is those classes that obstruct the decomposition. Those classes are natural with respect to the induced vector bundle by a map. For some special decompositions, we gave a relationship between those classes and the well-known characteristic classes such as Stiefel-Whitney classes and Chern classes. We determined the local coefficients in the cohomology group which contain the decomposition obstruction classes. We find applications in the study of subbundles of low codimension. In particular, codimension 1 decomposition classes are investigated in which we find that one of the two decomposition classes for the universal bundle over $B O(2 n+1)$ is in $H^{2 n+1}(B O(2 n+1), Z)$. This result gives rise to a new geometric interpretation for the order two elements in the integral cohomology group in odd dimension. We further make use of the cellular structure of the classifying space $B O(n)$ to see the 'local' structure for the restriction of the universal bundle to each cell. In this way, we can construct the obstruction classes for the codimension 1 vector bundle decomposition. We gave an example to calculate the decomposition obstruction for the tangent bundle of $R P^{2 n}$, which turns out to be the generator in the cohomology of $R P^{2 n}$ with twisted integer coefficients. On the other hand, we exhibit a trivial summand in the tangent bundle for any odd dimensional cobordism classes.

\section{Introduction.}

The classification for vector bundles is a classical problem which has been studied by many mathematicians. Grothendieck proved that every algebraic vector bundle over $C P^{1}$ can be decomposed as a direct sum of complex line bundles which gives rise to a complete classification for algebraic vector bundle over $C P^{1}$. Hirzebruch [6] applied the Riemann-Rock theorem to the vector bundles over $C P^{n}$ and obtained an integral condition on the Chern classes of the vector bundle. Using the Hirzebruch's results, Schwarzenberger 
[12] gave a partial classification for complex algebraic 2-vector bundles over $C P^{2}$ and formulated the following conditions for the Chern classes $c_{1}$ and $c_{2}$ of a 2-vector bundle over $C P^{n}$ :

$$
S:\left(\begin{array}{c}
\delta_{1} \\
k
\end{array}\right)+\left(\begin{array}{c}
\delta_{2} \\
k
\end{array}\right) \in Z \quad 2 \leq k \leq n
$$

where $\delta_{1}+\delta_{2}=c_{1}, \delta_{1} \delta_{2}=c_{2}$.

Atiyah and Rees [1] classified the complex topological 2-vector bundles over $C P^{3}$. In [2], Barth and Van de Ven gave a decomposability criterion for complex algebraic 2-bundles over $C P^{n}$. Switzer [14] contributed in the classification of complex topological 2-vector bundle over $C P^{n}$ for $n=4,5$ and 6 .

In the real case the literature is sketchy at best. In this paper we shall begin the study of the decomposition of real vector bundles. Unlike complex vector bundles, the decomposition problem for real vector bundles will involve cohomology groups with local coefficients. We give a general decomposition result (Theorem 2.1.5) which relates a given vector bundle to some cohomology classes with local coefficients in the homotopy group of a Grassmann manifold; it is those classes that obstruct the decomposition. Those classes are natural with respect to the induced vector bundle by a map (see 2.1.7). For some special decompositions, we gave a relationship between those classes and the well-known characteristic classes such as Stiefel-Whitney classes and Chern classes (see 2.2.8, 2.2.9 and 2.2.10). We find applications in the study of subbundles of low codimension. In particular, codimension 1 decomposition classes are investigated in 2.2.6 in which we find that one of the two decomposition classes for the universal bundle over $B O(2 n+1)$ is in $H^{2 n+1}(B O(2 n+1), Z)$. This result gives rise to a new geometric interpretation for the order two elements in the integral cohomology group in odd dimension. We further make use of the cellular structure of the classifying space $B O(n)$ to see the 'local' structure (see 2.2.11) for the restriction of the universal bundle to each cell. In this way, we can construct the obstruction classes for the codimension 1 vector bundle decomposition. In example 3.1, we calculated the decomposition obstruction for the tangent bundle of $R P^{2 n}$, which turns out to be the generator in the cohomology of $R P^{2 n}$ with twisted integer coefficients. On the other hand, in Example 3.4, we exhibit a trivial summand in the tangent bundle for any odd dimensional cobordism classes.

Our approach is based on the following considerations. Let $G$ be a compact Lie group and $H$ be a closed subgroup of $G$. Designated by $B G$ and $B H$ the classifying spaces of $G$ and $H$ respectively, classical results on compact Lie groups and their classifying spaces give us an important fibration $B H \longrightarrow B G$ with fiber $G / H[\mathbf{1 5}]$. The lifting problem for certain fibrations 
between the classifying spaces of classical Lie groups and their closed subgroups is an extensively studied problem during the past twenty years [7], [8], [11] and [9]. We will discuss the following fibration in some detail:

$$
B(O(n) \times O(m)) \longrightarrow B O(n+m) .
$$

The fibre of the fibration is $O(n+m) / O(n) \times O(m)=G_{n, m}$, the Grassmann manifold. Let $X$ be a CW-complex and $\xi^{n}$ be an $n$-dimensional vector bundle over $X$, then by classification theorem there is a continuous map, the classifying map of $\xi^{n}, f: X \longrightarrow B O(n)$ such that $\xi^{n} \approx f^{*}\left(\eta_{n}\right)$ where $\eta_{n}$ is the canonical $n$-dimensional vector bundle over $B O(n)$. A vector bundle $\xi^{n}$ can be decomposed into a Whitney sum $\xi^{n}=\xi^{k} \oplus \xi^{n-k}$ of two bundles if and only if the structure group of $\xi^{n}$ can be reduced into the subgroup $O(k) \times O(n-k)$ which means that the classifying map $f$ of $\xi^{n}$ can be lifted to the classifying space $B(O(k) \times O(n-k))$ up to a homotopy. So the problem of decomposing a vector bundle is equivalent to the lifting problem of its classifying map for the fibration $B(O(k) \times O(n-k)) \longrightarrow B O(n)$. We apply the obstruction theory to study the corresponding lifting problem for this fibration.

\section{Main results.}

2.1. The general decomposition results. In this section we will use the obstruction theory to consider some problems about vector bundles, in particular the decomposition problem of vector bundles over a CW-complex.

Lemma 2.1.1. Let $\xi^{m}$ be an $m$-dimensional vector bundle over a paracompact space $X$, then $\xi^{m}$ has a Whitney sum decomposition $\xi^{k} \oplus \xi^{m-k}$ if and only if there exists a commutative diagram up to homotopy:

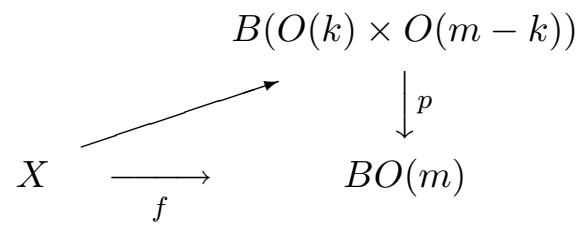

where $f$ is the classifying map of $\xi^{m}$ and $p$ is the map between the classifying spaces induced by the inclusion $O(k) \times O(m-k) \subset O(m)$.

Proof. This result can be proved by considering the structure group of a vector bundle.

There are different ways to construct the classifying space $B G$ for a compact Lie-group $G$. Here I give a geometric way to construct $B(O(k) \times O(m-$ $k)$ ) so that there is a natural fibration $p: B(O(k) \times O(m-k)) \longrightarrow B O(m)$ with fiber $G_{k}\left(R^{m}\right)$, the Grassmann. Let $\eta_{m}$ be the universal $m$-dimensional 
vector bundle over $B O(m)=G_{m}\left(R^{\infty}\right)$. Define

$$
\begin{aligned}
G_{k}\left(\eta_{m}\right) & =\{(X, Y) \mid Y \text { is any } k \text {-dimensional subspace of } X\} \\
& \subset G_{m}\left(R^{\infty}\right) \times G_{k}\left(R^{\infty}\right)
\end{aligned}
$$

with the subspace topology.

Proposition 2.1.2. $G_{k}\left(\eta_{m}\right)$ is a classifying space for $O(k) \times O(m-k)$. The natural projection $p: G_{k}\left(\eta_{m}\right) \longrightarrow G_{m}\left(R^{\infty}\right)$ is a fibration with fiber $G_{k}\left(R^{m}\right)$. The universal bundle over $G_{k}\left(\eta_{m}\right)$ is $p^{*}\left(\eta_{m}\right)$ which is isomorphic to $\omega_{k} \oplus \bar{\omega}_{m-k}$, whose total spaces are as follows:

$$
\begin{aligned}
E\left(\omega_{k}\right) & =\{((X, Y), \nu) \mid \nu \in Y\} \subset G_{k}\left(\eta_{m}\right) \times R^{\infty} ; \\
E\left(\bar{\omega}_{m-k}\right) & =\{((X, Y), \nu) \mid \nu \in X, \nu \perp Y\} \subset G_{k}\left(\eta_{m}\right) \times R^{\infty} .
\end{aligned}
$$

Proof. The proof is based on the uniqueness of the classifying space for compact Lie-group and the fact that the canonical inclusion $i: G_{k}\left(R^{\infty}\right) \times$ $G_{m-k}\left(R^{\infty}\right) \longrightarrow G_{m}\left(R^{\infty}\right)$ factors through $G_{k}\left(\eta_{m}\right)$.

By the local triviality of the universal bundle $\eta_{m}, G_{k}\left(\eta_{m}\right)$ is also a local trivial fiber space with fiber $G_{k}\left(R^{\infty}\right)$, therefore, the projection $p$ is a fibration with fiber $G_{k}\left(R^{m}\right)$.

Let $\xi^{m}$ be any $m$-dimensional vector bundle over a CW-complex $X$ with classifying map $f: X \longrightarrow G_{m}\left(R^{\infty}\right)$. We define $G_{k}\left(\xi^{m}\right) \longrightarrow X$, called the Grassmann bundle associated with $\xi^{m}$, to be the pull-back of the fibration $p: G_{k}\left(\eta_{m}\right) \longrightarrow G_{m}\left(R^{\infty}\right)$ by $f$. To justify the definition, we need to prove that if $f$ is homotopic to $g$, then their pull-backs must be homeomorphism. By the classification theorem, $f^{*}\left(\eta_{m}\right) \underset{h}{\approx} g^{*}\left(\eta_{m}\right) \approx \xi^{m}$. Now we can define a homeomorphism $h^{*}: f^{*}\left(G_{k}\left(\eta_{m}\right)\right) \approx g^{*}\left(G_{k}\left(\eta_{m}\right)\right)$ by

$$
h^{*}(x,(f(x), Y))=(x,(h(f(x)), h(Y)))=(x,(g(x), h(Y)))
$$

where $Y$ is any $k$-dimensional subspace of $f(x)$. Geometrically, $G_{k}\left(\xi^{m}\right)$ is the space of all $k$-dimensional subspaces in the fibers of $\eta_{m}$. As a special case, $G_{1}\left(\xi^{m}\right)$ is the well-known projective bundle space $R P\left(\xi^{m}\right)$ associated with $\xi^{m}$.

Lemma 2.1.3. The projection $p_{\xi}: G_{k}\left(\xi^{m}\right) \longrightarrow X$ is a fibration with fiber $G_{k}\left(R^{m}\right)$. $\xi^{m}$ has a decomposition $\xi^{m} \approx \xi_{1}^{k} \oplus \xi_{2}^{m-k}$ if and only if there is a section for the fibration $p_{\xi}$.

Proof. Let $f: X \longrightarrow G_{m}\left(R^{\infty}\right)$ be the classifying map for $\xi^{m}$, then we have a pull-back diagram:

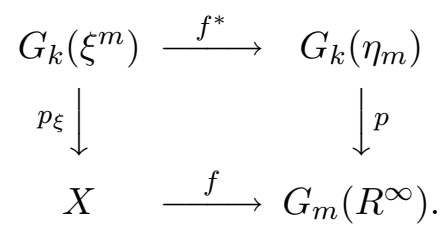


By 2.1.1 and 2.1.2 $\xi^{m} \approx \xi_{1}^{k} \oplus \xi_{2}^{m-k}$ if and only if there is a lifting for the fibration $p: G_{k}\left(\eta_{m}\right) \longrightarrow G_{m}\left(R^{\infty}\right)$ which is equivalent to the existence of a section of the fibration $p_{\xi}: G_{k}\left(\xi^{m}\right) \longrightarrow X$.

Let $p: E \longrightarrow B$ be a fibration with fiber $F$, and let $E, B$ and $F$ be pathconnected $\mathrm{CW}$-spaces. Then there exist fibrations $q_{n}$ and maps $h_{n}$ making the digram

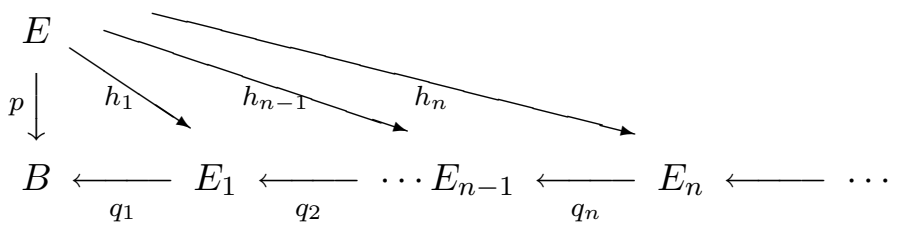

commute, and such that for $n>1$ (if $n=1, \pi_{1}(F)$ needs to be abelian):

(1) $q_{n}$ is a fibration with fiber $K\left(\pi_{n}(F), n\right)$, the Eilenberg-Maclane space.

(2) $h_{n}$ is $(n+1)$-connected.

In the following, we will use the above so-called Postnikov decomposition for a fibration to study $p: G_{k}\left(\eta_{m}\right) \longrightarrow G_{m}\left(R^{\infty}\right)$ or $p_{\xi}: G_{k}\left(\xi^{m}\right) \longrightarrow X$. We start with a typical fibration in the Postnikov decomposition.

Lemma 2.1.4. Let $p: E \longrightarrow B$ be a fibration with fiber $K(\Pi, n)$, where $\Pi$ is an abelian group, and $f: X \longrightarrow B$ be a map between connected $\mathrm{CW}$ spaces. Let $\widehat{\Pi}_{p}$ be the local coefficients and $\mathrm{ob}(p) \in H^{n+1}\left(B ; \widehat{\Pi}_{p}\right)$ be the primary cohomology obstruction with respect to the trivial section on the base point. Then $f^{*}(\mathrm{ob}(p))=0 \in H^{n+1}\left(X ; f^{*} \widehat{\Pi}_{p}\right)$ if and only if $f$ can be lifted to $\tilde{f}: X \longrightarrow E$.

Proof. See [3].

Now we can apply the above results to prove our general decomposition theorem.

Theorem 2.1.5. Let $\xi^{m}$ be an m-dimensional vector bundle over a connected $\mathrm{CW}$-complex $X, p_{\xi}: G_{k}\left(\xi^{m}\right) \longrightarrow X$ be the Grassmann bundle of $\xi^{m}$ with Postnikov decomposition $\left\{\widetilde{X}_{n}, \widetilde{h}_{n}, \widetilde{q}_{n}\right\}$. Let

$$
\mathrm{ob}_{n}^{k}\left(\xi^{m}\right) \in H^{n+1}\left(\widetilde{X}_{n-1}, \widetilde{\pi}_{n}\left(G_{m-k, k}\right)\right), \quad n=1,2, \ldots
$$

be the $n$-th Postnikov invariance for $p_{\xi}$. For $n \geq 1$ and a given homomorphism $\theta: \pi_{1}(X) \longrightarrow \pi_{1}\left(\widetilde{X}_{1}\right)$, set

$$
\begin{aligned}
& \mathrm{OB}_{n, \theta}^{k}\left(\xi^{m}\right)=\left\{s_{n-1}^{*}\left(\mathrm{ob}_{n}^{k}\left(\xi^{m}\right)\right) \mid\right. \text { for all sections } \\
& \qquad \begin{array}{r}
\left.s_{n-1}: X \longrightarrow \widetilde{X}_{n-1} \text { s.t. }\left(\widetilde{q}_{2} \ldots \widetilde{q}_{n-1} s_{n-1}\right)_{*}=\theta\right\} \\
\qquad H^{n+1}\left(X, \theta_{*} \widehat{\pi}_{n}\left(G_{m-k, k}\right)\right)
\end{array}
\end{aligned}
$$


where $s_{n-1}^{*}: H^{n+1}\left(\widetilde{X}_{n-1} ; \widehat{\pi}_{n}\left(G_{m-k, k}\right)\right) \longrightarrow H^{n+1}\left(X ; \theta_{*} \widehat{\pi}_{n}\left(G_{m-k, k}\right)\right)$ is the induced homomorphism in the cohomology groups with local coefficients. Then

$$
\left\{\mathrm{ob}_{n}^{k}\left(\xi^{m}\right) \mid n=1,2, \ldots\right\} \text { and }\left\{\mathrm{OB}_{n, \theta}^{k}\left(\xi^{m}\right) \mid n=1,2, \ldots\right\}
$$

have the following properties:

(1) If $\mathrm{ob}_{n}^{k}\left(\xi^{m}\right)=0$ for every $n<\operatorname{dim} X$, then $\xi^{m}$ can be decomposed as a Whitney sum $\xi^{m}=\xi^{k} \oplus \xi^{m-k}$.

(2) If $\xi^{m}$ can be decomposed as a Whitney sum $\xi^{m}=\xi^{k} \oplus \xi^{m-k}$, then there exists a homomorphism $\theta: \pi_{1}(X) \longrightarrow \pi_{1}\left(\widetilde{X}_{1}\right)$ such that $0 \in \mathrm{OB}_{n, \theta}^{k}\left(\xi^{m}\right)$ for $n=1,2, \ldots$.

(3) If $N=\operatorname{dim} X<\infty$ and $0 \in \mathrm{OB}_{N-1, \theta}^{k}\left(\xi^{m}\right)$, then $\xi^{m}$ can be decomposed as a Whitney sum $\xi^{m}=\xi^{k} \oplus \xi^{m-k}$.

(4) $\xi^{m}$ can be decomposed as a Whitney sum $\xi^{m}=\xi^{k} \oplus \xi^{m-k}$ if and only if there exists a section $s: X \longrightarrow \varliminf_{n} \widetilde{X}_{n}$ for the fibration pr: $\lim _{n} \tilde{X}_{n} \longrightarrow X$.

Proof. By Lemma 2.1.3, the problem of decomposition $\xi^{m}=\xi^{k} \oplus \xi^{m-k}$ is equivalent to the existence of a section for the fibration $p_{\xi}: G_{k}\left(\xi^{m}\right) \longrightarrow X$ which has fiber $G_{m-k, k}$.

If $k=1$, and $m=2$, then $G_{m-k, k}=G_{1,1}=S^{1}$ and $\pi_{1}\left(G_{1,1}\right)=Z$. If $m>2$, then it is well-known that

$$
\pi_{1}\left(G_{m-k, k}\right) \approx \pi_{0}(O(k))=Z_{2} .
$$

As a result, $\pi_{1}\left(G_{m-k, k}\right)$ is always an abelian group so that we can apply the obstruction theory for the fibration $p_{\xi}: G_{k}\left(\xi^{m}\right) \longrightarrow X$.

Proof of (1). By Lemma 2.1.4, $\mathrm{ob}_{n}^{k}\left(\xi^{m}\right)=0$ if and only if the fibration $\widetilde{q}_{n}: \widetilde{X}_{n} \longrightarrow \widetilde{X}_{n-1}$ has a section. So if $\mathrm{ob}_{n}^{k}\left(\xi^{m}\right)=0$ for each $n$, then there exists a section $s: X \longrightarrow \lim _{n} \widetilde{X}_{n}$ for the fibration $p r: \varliminf_{n} \widetilde{X}_{n} \longrightarrow$ $X$, which is the composition of all sections $\widetilde{q}_{n}: \widetilde{X}_{n} \longrightarrow \widetilde{X}_{n-1}$. From [3], $\varliminf_{n} \widetilde{h}_{n}: G_{k}\left(\xi^{m}\right) \longrightarrow \varliminf_{n} \widetilde{X}_{n}$ is a weak homotopy equivalence. Since $X$ is a CW-complex, one can apply J.H.C. Whitehead theorem, which in our situation says that $h^{*}:\left[X, G_{k}\left(\xi^{m}\right)\right] \approx\left[X, \lim _{n} \widetilde{X}_{n}\right]$, to get a section for the fibration $p_{\xi}: G_{k}\left(\xi^{m}\right) \longrightarrow X$. By Lemma 2.1.3, $\xi^{m}$ can be decomposed as a Whitney sum: $\xi^{m}=\xi^{k} \oplus \xi^{m-k}$.

Proof of (2). By Lemma 2.1.3, $\xi^{m}=\xi^{k} \oplus \xi^{m-k}$ implies $p_{\xi}: G_{k}\left(\xi^{m}\right) \longrightarrow X$ has a sections $s: X \longrightarrow G_{k}\left(\xi^{m}\right)$ which gives rise to a sequence of sections $\left\{s_{n}=h_{n} s: X \longrightarrow \widetilde{X}_{n} \mid\right.$ s.t. $\left.\widetilde{q}_{n} s_{n}=s_{n-1}, n=1,2, \ldots\right\}$. Take $\theta=s_{1 *}$ : $\pi_{1}(X) \longrightarrow \pi_{1}\left(\widetilde{X}_{1}\right)$. Then for every $n$, consider the pull-back diagram: 


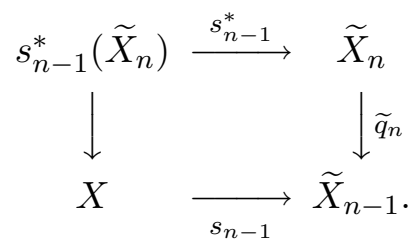

By definition, $\mathrm{ob}_{n}^{k}\left(\xi^{m}\right)$ is the primary cohomology obstruction for the fibration $\widetilde{q}_{n}: \widetilde{X}_{n} \longrightarrow \widetilde{X}_{n-1}$. Since $s_{n-1}$ has a lifting $s_{n}$, by Lemma 2.1.4,

$$
s_{n-1}^{*}\left(\mathrm{ob}_{n}^{k}\left(\xi^{m}\right)\right)=0 \in H^{n+1}\left(X ; s_{n-1 *} \widehat{\pi}_{n}\left(G_{m-k, k}\right)\right) .
$$

Now we look at the local coefficients. From the long exact sequence of the fibration $\widetilde{q}_{n}: \widetilde{X}_{n} \longrightarrow \widetilde{X}_{n-1}$ :

$$
\ldots \stackrel{\partial_{2}}{\longrightarrow} \pi_{1}\left(K\left(\pi_{n}(F), n\right)\right) \stackrel{i_{*}}{\longrightarrow} \pi_{1}\left(\widetilde{X}_{n}\right) \stackrel{\widetilde{q}_{n^{*}}}{\longrightarrow} \pi_{1}\left(\widetilde{X}_{n-1}\right) \longrightarrow 0
$$

we see that $\widetilde{q}_{n^{*}}: \pi_{1}\left(\widetilde{X}_{n}\right) \longrightarrow \pi_{1}\left(\widetilde{X}_{n-1}\right)$ is an isomorphism for $n \geq 2$. Noticing that

$$
\widetilde{q}_{2} \ldots \widetilde{q}_{n-1} s_{n-1}=s_{1},
$$

we thus prove that $s_{n-1 *} \widehat{\pi}_{n}\left(G_{k, m-k}\right)=\theta_{*} \widehat{\pi}_{n}\left(G_{k, m-k}\right)$, and

$$
s_{n-1}^{*}\left(\mathrm{ob}_{n}^{k}\left(\xi^{m}\right)\right)=0 \text { in } H^{n+1}\left(X ; \theta_{*} \widehat{\pi}_{n}\left(G_{m-k, k}\right)\right) .
$$

By definition, $0 \in \mathrm{OB}_{n, \theta}^{k}\left(\xi^{m}\right)$, for $n=1,2, \ldots$.

Proof of (3). By definition, $0 \in \mathrm{OB}_{N-1, \theta}^{k}\left(\xi^{m}\right)$ implies that there exists a section $s_{N-2}$ such that $s_{N-2}^{*}\left(\mathrm{ob}_{N-1}\left(\xi^{m}\right)\right)=0$ in $H^{N}\left(X, \theta_{*} \widehat{\pi}_{N-1}\left(G_{m-k, k}\right)\right)$, by Lemma 2.1.4, $s_{N-2}$ has a lifting $s_{N-1}$ such that $p_{N-1} s_{N-1}=s_{N-2}$. Since $\operatorname{dim} X=N$, for any local coefficients $\widetilde{G}$ on $X$

$$
H^{i}(X, \widetilde{G})=0 \quad \text { for } i>N .
$$

But $s_{N-1}^{*}\left(\operatorname{ob}_{N}\left(\xi^{m}\right)\right) \in H^{N+1}\left(X, s_{N-1 *} \widehat{\pi}_{N}\left(G_{k, m-k}\right)\right)=0$, so $s_{N-1}^{*}\left(\operatorname{ob}_{N}\left(\xi^{m}\right)\right)$ $=0$, and by Lemma 2.1.4, there exists a section $s_{N}$ such that $p_{N} s_{N}=$ $s_{N-1}$. By repeating this procedure, we can obtain a sequence of sections $\left\{s_{n} \mid\right.$ s.t. $\left.p_{n} s_{n}=s_{n-1}\right\}$ which gives a section for the fibration $p_{\xi}: G_{k}\left(\xi^{m}\right) \longrightarrow$ $X$ and by Lemma 2.1.3, $\xi^{m}=\xi^{k} \oplus \xi^{m-k}$.

Proof of (4). Since $\lim _{n} \widetilde{h}_{n}: G_{k}\left(\xi^{m}\right) \longrightarrow \varliminf_{n} \widetilde{X}_{n}$ is a weak homotopy equivalence and $X$ is a CW-complex, from Lemma 2.1.1 and J.H.C. Whitehead theorem as in proof of (1) the result follows.

Definition 2.1.6. The classes $\left\{\mathrm{ob}_{n}^{k}\left(\xi^{m}\right) \in H^{n+1}\left(\widetilde{X}_{n-1}, \widehat{\pi}_{n}\left(G_{m-k, k}\right)\right), n=\right.$ $1,2 \ldots\}$ in the above theorem are called the decomposition obstructions of $\xi^{m}=\xi^{k} \oplus \xi^{m-k}$. 
Corollary 2.1.7. $\left\{\mathrm{ob}_{n}^{k}\left(\xi^{m}\right) \in H^{n+1}\left(\widetilde{X}_{n-1}, \widehat{\pi}_{n}\left(G_{m-k, k}\right)\right), n=1,2 \ldots\right\}$ are natural in the following sense: If $g: Y \longrightarrow X$ is a map, then $g$ pulls the tower

$$
X \longleftarrow \widetilde{q}_{1} \widetilde{X}_{1} \longleftarrow \cdots \widetilde{X}_{n-1} \longleftarrow \widetilde{q}_{n} \tilde{X}_{n} \longleftarrow \cdots
$$

back over $Y$ such that

$$
g_{n-1}^{*}\left(\mathrm{ob}_{n}^{k}\left(\xi^{m}\right)\right)=\mathrm{ob}_{n}^{k}\left(g^{*}\left(\xi^{m}\right)\right) \text { and }
$$

$$
g^{*}\left(\mathrm{OB}_{n \theta}^{k}\left(\xi^{m}\right)\right) \subset \mathrm{OB}_{n, g_{*} \theta}^{k}\left(g^{*}\left(\xi^{m}\right)\right)
$$

where $g_{n-1}: g^{*}(\widetilde{X})_{n-1} \longrightarrow \widetilde{X}_{n-1}$ is the induced map at $(n-1)$ stage, in particular,

$$
\begin{aligned}
& \mathrm{ob}_{n}^{k}\left(\xi^{m}\right)=\mathrm{ob}_{n}^{k}\left(f^{*}\left(\eta_{m}\right)\right)=f_{n-1}^{*}\left(\mathrm{ob}_{n}^{k}\left(\eta_{m}\right)\right) \text { and } \\
& \qquad f^{*}\left(\mathrm{OB}_{n, \theta}^{k}\left(\eta_{m}\right)\right) \subset \mathrm{OB}_{n, f_{*} \theta}^{k}\left(\xi^{m}\right)
\end{aligned}
$$

where $\eta_{m}$ is the universal m-vector bundle, and $f$ is the classifying map of $\xi^{m}$.

Proof. The proof is essentially based on the naturality of the primary cohomology obstruction and that of the Postnikov decomposition. By definition $\mathrm{ob}_{n}^{k}\left(\xi^{m}\right)$ is the $n$-th Postnikov invariant in the induced Postnikov decomposition. But the Postnikov invariants are natural since they are defined to be the primary cohomology obstructions.

To prove that $g^{*}\left(\mathrm{OB}_{n \theta}^{k}\left(\xi^{m}\right)\right) \subset \mathrm{OB}_{n, g_{*} \theta}^{k}\left(g^{*}\left(\xi^{m}\right)\right)$, let $s_{n-1}: X \longrightarrow \widetilde{X}_{n-1}$ be a section such that $\left(\widetilde{q}_{2} \cdots \widetilde{q}_{n-1} s_{n-1}\right)_{*}=\theta$, consider the commutative diagram:

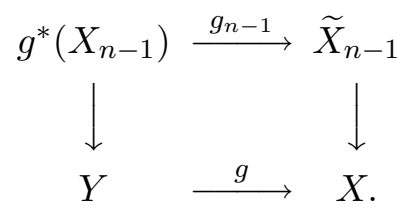

Since $s_{n-1}$ induces a section $g^{*}\left(s_{n-1}\right)$ for the induced fibration by $g$ such that $s_{n-1} g=g_{n-1} g^{*}\left(s_{n-1}\right)$, by the naturality of cohomology with local coefficients, we have

$$
\begin{aligned}
g^{*}\left(s_{n-1}^{*}\left(\mathrm{ob}_{n}^{k}\left(\xi^{m}\right)\right)\right) & =\left(s_{n-1} g\right)^{*}\left(\mathrm{ob}_{n}^{k}\left(\xi^{m}\right)\right) \\
& =\left(g_{n-1} g^{*}\left(s_{n-1}\right)\right)^{*}\left(\mathrm{ob}_{n}^{k}\left(\xi^{m}\right)\right) \\
& =\left(g^{*}\left(s_{n-1}\right)\right)^{*} g_{n-1}^{*}\left(\mathrm{ob}_{n}^{k}\left(\xi^{m}\right)\right) \\
& =\left(g^{*}\left(s_{n-1}\right)\right)^{*}\left(\mathrm{ob}_{n}^{k}\left(g^{*}\left(\xi^{m}\right)\right)\right)
\end{aligned}
$$

which means that $g^{*}\left(\mathrm{OB}^{k, n, \theta}\left(\xi^{m}\right)\right) \subset \mathrm{OB}_{n, g_{*} \theta}^{k}\left(g^{*}\left(\xi^{m}\right)\right)$. 
In the following we study the decomposition obstructions in some details. Consider the inclusions:

$$
O(m-k) \subset O(m-k) \times O(k) \subset O(m)
$$

which induce fibrations in their classifying spaces:

$$
B O(m-k) \longrightarrow B(O(m-k) \times O(k)) \longrightarrow B O(m) .
$$

We look at the following two fibrations:

$$
p: B O(m-k) \longrightarrow B O(m) \text { and } p^{\prime}: B(O(m-k) \times O(k)) \longrightarrow B O(m)
$$

which have fibers $O(m) / O(m-k)=V_{m, k}$, and $O(m) /(O(m-k) \times O(k))=$ $G_{m-k, k}$ respectively, and view the third fibration $B O(m-k) \longrightarrow B(O(m-$ $k) \times O(k))$ as a map between the two fibrations. By the naturality of Postnikov decomposition, there exists a commutative diagram:

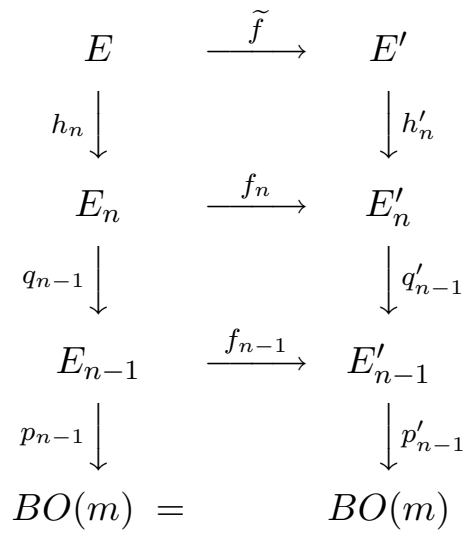

where $\left\{E_{n}, . q_{n}, h_{n}\right\}$ and $\left\{E_{n}^{\prime}, . q_{n}^{\prime}, h_{n}^{\prime}\right\}$ are the Postnikov decompositions of $p: B O(m-k) \longrightarrow B O(m)$ and $p^{\prime}: B(O(m-k) \times O(k)) \longrightarrow B O(m)$ respectively.

Now we study the Postnikov decomposition $\left\{E_{n}, . q_{n}, h_{n}\right\}$ of $p: B O(m-$ $k) \longrightarrow B O(m)$. Since $q_{n}$ is a fibration with fiber $K\left(\pi_{n}\left(V_{m, k}\right), n\right)$ and the Stiefel manifold $V_{m, k}$ is $(m-k-1)$-connected, the Postnikov invariant

$$
k_{i}(p) \in H^{i+1}\left(E_{i-1} ; \widehat{\pi}_{i}\left(V_{m, k}\right)\right)
$$

vanishes for $0<i<m-k$. Applying Lemma 2.1.4 repeatedly, we have:

Proposition 2.1.8. There exists a section: $B O(m) \longrightarrow E_{m-k-1}$ in the Postnikov decomposition $\left\{E_{n}, q_{n}, h_{n}\right\}$ of the fibration $p: B O(m-k) \longrightarrow$ $B O(m)$.

Corollary 2.1.9. There exists a section: $B O(m) \longrightarrow E_{m-k-1}^{\prime}$ in the Postnikov decomposition $\left\{E_{n}^{\prime}, q_{n}^{\prime}, h_{n}^{\prime}\right\}$ of the fibration $p^{\prime}: B(O(m-k) \times O(k)) \longrightarrow$ $B O(m)$. 
Proof. This is a direct result of the Proposition 2.1.8 and the above commutative diagram.

Corollary 2.1.10. Let $\xi^{m}$ be an m-dimensional vector bundle over a connected $N$-dimensional $\mathrm{CW}$-complex $X$. If $N \leq m-k$, then $\xi^{m}$ can be decomposed as a Whitney sum $\xi^{m}=\xi^{k} \oplus \xi^{m-k}$.

Proof. By Corollary 2.1.9, we have a section: $s: B O(m) \longrightarrow E_{m-k-1}^{\prime}$ in the Postnikov decomposition $\left\{E_{n}^{\prime}, q_{n}^{\prime}, h_{n}^{\prime}\right\}$ of the fibration $p^{\prime}: B O((m-k) \times$ $O(k)) \longrightarrow B O(m)$. So there exists a section: $\widetilde{s}: X \longrightarrow \widetilde{X}_{m-k-1}$ in the induced Postnikov decomposition. By definition, $0 \in \mathrm{OB}_{m-k-1, \theta}^{k}\left(\xi^{m}\right)$, using Theorem 2.1.5 (3), one concludes that $\xi^{m}$ can be decomposed as a Whitney $\operatorname{sum} \xi^{m}=\xi^{k} \oplus \xi^{m-k}$.

In Theorem 2.1.5, the obstruction set $\mathrm{OB}_{n, \theta}^{k}\left(\xi^{m}\right)$ depends on $\theta: \pi_{1}(X) \rightarrow$ $\pi_{1}\left(\widetilde{X}_{1}\right)$. In the following, we will see that there are exactly two different $\theta$ 's for the universal $m$-vector bundle $\eta^{m}$.

Theorem 2.1.11. In the Postnikov decomposition $\left\{E_{n}^{\prime}, q_{n}^{\prime}, h_{n}^{\prime}\right\}$ of the fibration $p^{\prime}: B O((m-k) \times O(k)) \longrightarrow B O(m)$, if $m>2$, then there are exactly two homomorphisms

$$
\theta_{1}, \theta_{2}: \pi_{1}(B O(m)) \longrightarrow \pi_{1}\left(E_{1}^{\prime}\right)
$$

which are induced by some sections from $B O(m)$ to $E_{1}^{\prime}$.

Proof. Recall that in the Postnikov decomposition $\left\{E_{n}^{\prime}, q_{n}^{\prime}, h_{n}^{\prime}\right\}, q_{1}^{\prime}: E_{1}^{\prime} \longrightarrow$ $B O(m)$ is a fibration with fiber $K\left(\pi_{1}\left(G_{m-k, k}\right), 1\right)$. Consider the homotopy sequence of the fibration

$$
\begin{aligned}
& \cdots \longrightarrow \pi_{2}(B O(m)) \stackrel{\partial}{\longrightarrow} \pi_{1}\left(K\left(\pi_{1}\left(G_{m-k, k}\right), 1\right)\right) \\
& \qquad \stackrel{i_{*}}{\longrightarrow} \pi_{1}\left(E_{1}^{\prime}\right) \stackrel{q_{1 *}^{\prime}}{\longrightarrow} \pi_{1}(B O(m)) \longrightarrow 0
\end{aligned}
$$

and the fact that $\pi_{1}\left(G_{m-k, k}\right)=Z_{2}$ for $m>2$ and $\pi_{1}(B O(m))=Z_{2}$. Since $B(O(m-k) \times O(k)) \sim B O(m-k) \times B O(k)$, we have

$$
\pi_{1}(B(O(m-k) \times O(k))) \approx \pi_{1}(B O(m-k)) \oplus \pi_{1}(B O(m-k)) \approx Z_{2} \oplus Z_{2} .
$$

But $h^{\prime}: B(O(m-k) \times O(k)) \longrightarrow E_{1}^{\prime}$ is 2-connected, so

$$
h_{1^{*}}^{\prime}: \pi_{1}(B(O(m-k) \times O(k))) \longrightarrow \pi_{1}\left(E_{1}^{\prime}\right)
$$

is an isomorphism and hence $\pi_{1}\left(E_{1}^{\prime}\right) \approx Z_{2} \oplus Z_{2}$. Therefore, the above exact sequence actually is the following sequence:

$$
\pi_{2}(B O(m)) \stackrel{\partial}{\longrightarrow} Z_{2} \stackrel{i_{*}}{\longrightarrow} Z_{2} \oplus Z_{2} \stackrel{q_{1 *}^{\prime}}{\longrightarrow} Z_{2} \longrightarrow 0 .
$$

By the exactness, the image of $\partial$ must be 0 , and we get a short exact sequence:

$$
0 \longrightarrow Z_{2} \stackrel{i_{*}}{\longrightarrow} Z_{2} \oplus Z_{2} \stackrel{p_{*}^{\prime}}{\longrightarrow} Z_{2} \longrightarrow 0
$$


It is easy to see that there are exactly two homomorphisms

$$
\theta_{1}, \theta_{2}: Z_{2} \longrightarrow Z_{2} \oplus Z_{2}
$$

which satisfy the condition: $p_{*}^{\prime} \circ \theta_{1}=p_{*}^{\prime} \circ \theta_{2}=1$. By the 2-extendability thorem [3], we get two sections: $s_{1}, s_{2}: B O(m) \longrightarrow E_{1}^{\prime}$ such that

$$
s_{i^{*}}=\theta_{i}: \pi_{1}(B O(m))=Z_{2} \longrightarrow \pi_{1}\left(E_{1}^{\prime}\right)=Z_{2} \oplus Z_{2} \quad \text { for } i=1,2 .
$$

This is what we need to prove.

2.2. Codimension 1 decomposition. Now we consider two special cases in which there is only one decomposition obstruction. The first one is that the dimension of the vector bundle is 2 . The other one is the case in which the codimension is 1 .

Proposition 2.2.1. For any two dimensional vector bundle $\xi^{2}$ over a connected $\mathrm{CW}$-complex $X$, there is only one decomposition obstruction

$$
\mathrm{ob}_{1}\left(\xi^{2}\right) \in H^{2}(X ; f * \widetilde{Z})
$$

such that $\mathrm{ob}_{1}\left(\xi^{2}\right)=0$ if and only if $\xi^{2}$ can be decomposed as $\xi^{2}=\xi^{1} \oplus \eta^{1}$, where $f: X \longrightarrow B O(2)$ is the classifying map of $\xi^{2}$, and $\widetilde{Z}$ is the twisted integer.

Proof. In the fibration $p: B(O(1) \times O(1)) \longrightarrow B O(2)$, the fiber is

$$
O(2) /(O(1) \times O(1)) \approx G_{1,1} \approx S^{1} \approx K(Z, 1),
$$

so the fibration itself is the Postnikov decomposition, and

$$
\mathrm{ob}_{n}\left(\xi^{2}\right)=0 \quad \text { for } n>1 .
$$

Since $\mathrm{ob}_{1}\left(\xi^{2}\right)$ is the only none trivial decomposition obstruction, by Theorem 2.1.5, ob ob $_{1}\left(\xi^{2}\right)=0$ if and only if $\xi^{2}$ can be decomposed as $\xi^{2}=$ $\xi^{1} \oplus \eta^{1}$.

Corollary 2.2.2. Let $\eta_{2}$ be the universal principal $O(2)$-bundle, then for any two dimensional vector bundle $\xi^{2}$ over a connected $\mathrm{CW}$-complex $X$ with classifying map $f$,

$$
f^{*}\left(\mathrm{ob}_{1}\left(\eta_{2}\right)\right)=0 \text { if and only if } \xi^{2} \text { can be deconposed as } \xi^{2}=\xi^{1} \oplus \eta^{1} \text {. }
$$

Proof. By the naturality of the decomposition obstruction

$$
f^{*}\left(\mathrm{ob}_{1}\left(\eta_{2}\right)\right)=\mathrm{ob}_{1}\left(f^{*}\left(\eta_{2}\right)\right)=\mathrm{ob}_{1}\left(\xi^{2}\right) .
$$

From Proposition 2.2.1, we have this corollary.

Now we turn to consider the second special case where the dimension of the vector bundle is same as that of the base space. The decomposition is such that one of the bundles in the sum is a line bundle.

In our definition of decomposition obstructions $\mathrm{ob}_{n}^{k}\left(\xi^{m}\right)$, we use the Postnikov decomposition induced by the classifying map of the vector bundle $\xi^{m}$ 
from the fibration $p: B(O(k) \times O(m-k)) \longrightarrow B O(m)$. The advantage of this approach is that those decomposition obstruction classes $\mathrm{ob}_{n}^{k}\left(\xi^{m}\right)$ only depend on the vector bundle $\xi^{m}$ and are natural in the sense as indicated in Corollary 2.1.7. If one has a section $s$ to the $(n-1)$-level in the induced Postnikov decomposition, then the vanishing of $s^{*}\left(\mathrm{ob}_{n}^{k}\left(\xi^{m}\right)\right)$ is equivalent to the existence of the $n$-level lifting for $s$. So the class $s^{*}\left(\mathrm{ob}_{n}^{k}\left(\xi^{m}\right)\right)$ has the similar property as that of the obstruction cohomology class for a section. On the other hand, as stated in [3], the construction for the Postnikov decomposition is much more difficult than that of $\mathrm{CW}$-decomposition. In some special cases, one may give a detail description of the Postnikov decomposition [14]. However, Eckmann and Hilton [5] showed that the cohomology obstructions for Postnikov and CW-decompositions are equivalent. To say precisely, let $(X, A)$ be a relative $\mathrm{CW}$-complex with $\mathrm{CW}$-decomposition $X=\lim _{n} X_{n}$, and let $p: E \longrightarrow X$ be a fibration with Postnikov decomposition $\left\{E_{n}, q_{n}, h_{n}\right\}$, then there is a bijection:

$$
\lambda:\left\langle X_{n}, E\right\rangle^{u, \theta} \mid X_{n-1} \approx\left\langle X, E_{n-1}\right\rangle^{\theta^{\prime}}
$$

where $u: A \longrightarrow p^{-1}(A)=E_{-1}$ is a partial section, $\theta: \pi_{1}(X) \longrightarrow \pi_{1}(E)$ is a splitting of $p$ (see [3]) and $\theta^{\prime}=h_{1^{*}} \circ \theta: \pi_{1}(X) \longrightarrow \pi_{1}\left(E_{1}\right)$, and $\left\langle X_{n}, E\right\rangle^{u, \theta}$ denoted the section homotopy classes relative to $u$ and compatible with $\theta$, and $\left\langle X_{n}, E\right\rangle^{u, \theta} \mid X_{n-1}$ is the set of all the restrictions on $X_{n-1}$. The bijection mapping $\lambda$ is given in the following way:

Let $\phi_{n} \in\left\langle X_{n}, E\right\rangle^{u, \theta}$, then $h_{n-1} \phi_{n} \mid X_{n-1}: X_{n-1} \longrightarrow E_{n-1}$ has an extension $h_{n-1} \phi_{n}: X_{n} \longrightarrow E_{n-1}$. But the fiber of the fibration: $E_{n-1} \longrightarrow X$ has no non-vanishing homotopy groups in dimension greater than $n-1$, so the section $h_{n-1} \phi_{n}: X_{n} \longrightarrow E_{n-1}$ can be extended to a section over $X$ which is defined to be $\lambda\left(\phi_{n} \mid X_{n-1}\right)$. From [3], this is well-defined bijection. Under this bijection, Eckmann and Hilton's result says that

$$
\left[\mathrm{ob}_{n}\left(\phi_{n}\right)\right]=\phi^{n-1} *\left(k_{n}\left(q_{n}\right)\right) \in H^{n+1}\left(X, A ; \theta * \pi_{n}(F)\right)
$$

where $\phi^{n-1}=\lambda\left(\phi_{n} \mid X_{n-1}\right)$, and $k_{n}\left(q_{n}\right) \in H^{n+1}\left(E_{n-1} ; \pi_{n}(F)_{q_{n}}\right)$ is the $n$-th Postnikov invariant for the fibration $q_{n}: E_{n} \longrightarrow E_{n-1}$.

Using the above result, we see that from the Postnikov invariants, one can recover all the obstructions classes defined by using the CW-decomposition of the base space. It is because of this, we can compute the obstruction $\mathrm{OB}_{n \theta}^{k}\left(\xi^{m}\right)$ without knowing the Postnikov decomposition. This makes it possible to compute $\mathrm{OB}_{n \theta}^{k}\left(\xi^{m}\right)$ by using only the $\mathrm{CW}$-decomposition of the base space.

In order to actually compute $\mathrm{OB}_{n \theta}^{k}\left(\xi^{m}\right) \subset H^{n+1}\left(X, \theta_{*} \widehat{\pi}_{n}\left(G_{m-k, k}\right)\right)$, one still needs to know the local coefficients $\widehat{\pi}_{n}\left(G_{m-k, k}\right)$. By the naturality 2.1.7, we know that $f^{*}\left(\mathrm{OB}_{n, \theta}^{k}\left(\eta_{m}\right)\right) \subset \mathrm{OB}_{n, f_{*} \theta}^{k}\left(\xi^{m}\right)$, where $f$ is the classifying map for the vector bundle $\xi^{m}$, and $\eta_{m}$ is the universal $m$-plane bundle over $B O(m)$. So it will be enough to determine the local coefficients for the 
fibration $p: B(O(k) \times O(m-k)) \longrightarrow B O(m)$ which is difficult in general because one does not know $\pi_{n}\left(G_{k, m-k}\right)$ for each $n$. In order to relate this coefficients to some known coefficients, we need the following lemma:

Lemma 2.2.3. Let $E_{2} \stackrel{q_{2}}{\longrightarrow} E_{1} \stackrel{q_{1}}{\longrightarrow} X$ be a tower of fibrations, and let $F_{2}$ and $F_{1}$ be the fibers of $q_{1} q_{2}$ and $q_{1}$ respectively, then for each $n$, the induced homomorphism $q_{2^{*}}: \pi_{n}\left(F_{2}\right) \longrightarrow \pi_{n}\left(F_{1}\right)$ is a homorphism $q_{2^{*}}$ : $\pi_{n}\left(F_{2}\right)^{q_{1} q_{2}} \longrightarrow \pi_{n}\left(F_{1}\right)^{q_{1}}$ between the two systems of local coefficients determined by the fibrations $q_{1} q_{2}$ and $q_{1}$.

Proof. Let $\widehat{E}_{2}, \widehat{E_{2}}$, be the mapping cylinders of $q_{1} q_{2}$ and $q_{1}$ respectively, then $q_{2}$ induces an obvious map $\widehat{q}_{2}:\left(\widehat{E}_{2}, E_{2}\right) \longrightarrow\left(\widehat{E}_{1}, E_{1}\right)$ which induces operator homomorphisms between the two homotopy exact sequences:

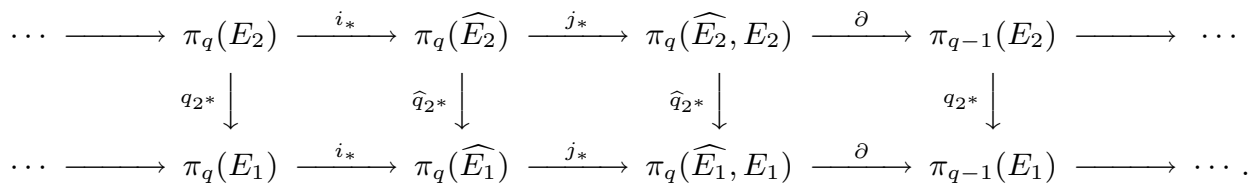

In particular, $\widehat{q}_{2^{*}}: \pi_{q}\left(\widehat{E}_{2}, E_{2}\right) \longrightarrow \pi_{q}\left(\widehat{E}_{1}, E_{1}\right)$ is an operator homorphism. Recall that $\pi_{n}\left(F_{1}\right)^{q_{1}}$ is defined via an isomorphism: $\Delta^{\prime}: \pi_{q}\left(\widehat{E_{1}}, E_{1}\right) \longrightarrow$ $\pi_{q-1}\left(F_{1}\right)$. In fact, the isomorphism is given by the following composition:

$$
\Delta^{\prime}: \pi_{q}\left(\widehat{E_{1}}, E_{1}\right) \stackrel{k_{1}^{-1}}{\longrightarrow} \pi_{q}\left(\widehat{F_{1}}, F_{1}\right) \stackrel{\partial}{\longrightarrow} \pi_{q-1}\left(F_{1}\right)
$$

where $k_{1}$ is the inclusion which induces an isomorphism [15]. Thus we have a commutative diagram:



Therefore $q_{2^{*}}: \pi_{n}\left(F_{2}\right)^{q_{1} q_{2}} \longrightarrow \pi_{n}\left(F_{1}\right)^{q_{1}}$ is a homomorphism between the two local systems.

Corollary 2.2.4. With the above notations, for any $n$-skeleton section $s$ : $X^{n} \longrightarrow E_{2}(n \geq 2)$, there is an induced homomorphism:

$$
q_{2_{*}}: H^{n+1}\left(X ; s_{*} \pi_{n}\left(F_{2}\right)\right) \longrightarrow H^{n+1}\left(X ; q_{2} \circ s_{*} \pi_{n}\left(F_{1}\right)\right)
$$

such that

$$
q_{2 *}\left(\mathrm{ob}_{n}(s)\right)=\mathrm{ob}_{n}\left(q_{2} \circ s\right) .
$$


Proof. By Lemma 2.2.3, $q_{2_{*}}: \pi_{n}\left(F_{2}\right) \longrightarrow \pi_{n}\left(F_{1}\right)$ is a homomorphism $q_{2_{*}}$ : $\pi_{n}\left(F_{2}\right)^{q_{1} q_{2}} \longrightarrow \pi_{n}\left(F_{1}\right)^{q_{1}}$ between the two systems of local coefficients determined by the fibrations $q_{1} q_{2}$ and $q_{1}$. The sections $s: X^{n} \longrightarrow E_{2}$ and $q_{2}$ $s: X^{n} \longrightarrow E_{1}$ pull the two systems of local coefficients back on $X^{n}$ so that $q_{2 *}: \pi_{n}\left(F_{2}\right)^{q_{1} q_{2}} \longrightarrow \pi_{n}\left(F_{1}\right)^{q_{1}}$ induces a homomorphism of the systems of local coefficients:

$$
q_{2 *}: s_{*} \pi_{n}\left(F_{2}\right) \longrightarrow\left(q_{2} s\right)_{*} \pi_{n}\left(F_{1}\right) .
$$

From [15], $q_{2 *}$ induces a homomorphism for the cohomology groups with local coefficients:

$$
q_{2_{*}}: H^{n+1}\left(X ; s_{*} \pi_{n}\left(F_{2}\right)\right) \longrightarrow H^{n+1}\left(X ; q_{2} \circ s_{*} \pi_{n}\left(F_{1}\right)\right) .
$$

Now we consider the diagram:

$$
\begin{array}{rccc}
\pi_{n+1}\left(E_{2}^{n+1}, E_{2}^{n}\right) & \partial & \pi_{n}\left(E_{2}^{n}\right) \stackrel{s_{\#}}{\longrightarrow} & \pi_{n}\left(F_{2}\right) \\
q_{2 *} \downarrow & q_{2 *} \downarrow & & q_{2 *} \downarrow \\
\pi_{n+1}\left(E_{1}^{n+1}, E_{1}^{n}\right) \stackrel{\partial}{\longrightarrow} & \pi_{n}\left(E_{1}^{n}\right) \stackrel{\left(q_{2} s\right)_{\#}}{\longrightarrow} & \pi_{n}\left(F_{1}\right)
\end{array}
$$

where all the spaces and homomorphisms are the same as in the definition of obstruction cocycle (see [3]). We only need to check the commutativity of the right square.

Let $\alpha \in \pi_{n}\left(E_{2}^{n}\right)$, by definition, we have

$$
\begin{aligned}
i_{*} q_{2 *} s_{\#}(\alpha) & =q_{2 *} i_{*} s_{\#}(\alpha)=q_{2 *}\left(\alpha-s_{*}\left(q_{1} q_{2}\right)_{*}(\alpha)\right) \\
& =q_{2 *}(\alpha)-q_{2 *} s_{*}\left(q_{1} q_{2}\right)_{*}(\alpha) \\
& =q_{2 *}(\alpha)-\left(q_{2} s\right)_{*}\left(q_{1}\left(q_{2 *}(\alpha)\right)\right)=i_{*}\left(q_{2} \circ s\right)_{\#} q_{2 *}(\alpha) .
\end{aligned}
$$

Since $i_{*}$ is injective, we obtain that $q_{2 *} s_{\#}=\left(q_{2} \circ s\right)_{\#} q_{2 *}$. Therefore, we have

$$
q_{2 *} s_{\#} \partial q_{2 *}^{-1}=\left(q_{2} \circ s\right)_{\#} \partial .
$$

By the definition of cocycle, and that of the induced homomorphism,

$$
q_{2 *}\left(\mathrm{ob}_{n}(s)\right)=\mathrm{ob}_{n}\left(q_{2} \circ s\right) .
$$

This completes the proof.

Corollary 2.2.5. The inclusions $O(m) \subset O(m) \times O(n) \subset O(m+n)$ induce a tower of fibrations $B O(m) \stackrel{q_{2}}{\longrightarrow} B(O(m) \times O(n)) \stackrel{q_{1}}{\longrightarrow} B O(m+n)$. Let $m \geq n$ and $m+n>2$. Then $q_{2}$ induces a homomorphism:

$q_{2 *}: H^{q+1}\left(B O(m+n) ; \pi_{q}\left(V_{m+n, n}\right)_{q_{1} q_{2}}\right) \longrightarrow H^{q+1}\left(B O(m+n) ; \theta_{1_{*}} \pi_{q}\left(G_{m, n}\right)\right)$ where $\theta_{1}: \pi_{1}(B O(m+n))=\pi_{1}(B O(m))=Z_{2} \stackrel{q_{2 *}}{\longrightarrow} \pi_{1}(B(O(m) \times O(n)))=$ $Z_{2} \oplus Z_{2}$. If $s$ is a q-skeleton section with $q \geq 2$, then

$$
q_{2 *}\left(\mathrm{ob}_{q}(s)\right)=\mathrm{ob}_{q}\left(q_{2} \circ s\right) .
$$


Proof. The fibers of $q_{1}$ and $q_{1} q_{2}$ are $G_{m, n}$ and $V_{m+n, n}$ respectively. By our assumption, $m \geq 2$, so $V_{m+n, n}$ is at least 1-connected and the fibration $q_{1} q_{2}$ has a unique (up to homotopy) 2-skeleton section $s$ and

$$
s_{*}: \pi_{1}(B O(m+n))=Z_{2} \longrightarrow \pi_{1}(B O(m))=Z_{2}
$$

is an isomorphism. The result follows by Corollary 2.2.4 if we simply think $s_{*}$ as an identity.

The following theorem is the main result about the codimension 1 decomposition which reveals an obstruction class in $H^{2 n+1}(B O(2 n+1) ; Z)$, the cohomology with ordinary integer coefficients.

Theorem 2.2.6. Let $\eta_{m}$ be the $m$-dimensional universal bundle over $B O(m)$ with $m \geq 3$, then there are exactly two obstruction classes in dimension $m$ for the decomposition $\eta_{m} \approx \xi^{m-1} \oplus \lambda$. Furthermore, one of the two classes is the primary obstruction for the decomposition $\eta_{m} \approx \xi^{m-1} \oplus R$, which is in $H^{m}(B O(m) ; \widetilde{Z})$, where $\widetilde{Z}$ is the twisted integers; the other is in $H^{m}(B O(m) ; \widetilde{Z})$ if $m$ is even and is in $H^{m}(B O(m) ; Z)$ if $m$ is odd.

Proof. Consider the tower of fibrations:

$$
B O(m-1) \stackrel{q_{2}}{\longrightarrow} B(O(m-1) \times O(1)) \stackrel{q_{1}}{\longrightarrow} B O(m)
$$

by Corollary 2.2.5, $q_{2}$ induces a homomorphism:

$$
q_{2 *}: H^{q+1}\left(B O(m) ; \pi_{q}\left(V_{m, 1}\right)_{q_{1} q_{2}}\right) \longrightarrow H^{q+1}\left(B O(m) ; \theta_{1_{*}} \pi_{q}\left(G_{m-1,1}\right)\right)
$$

where

$$
\begin{aligned}
\theta_{1}: \pi_{1}(B O(m)) & =\pi_{1}(B O(m-1)) \\
& =Z_{2} \stackrel{q_{2 *}}{\longrightarrow} \pi_{1}(B(O(m-1) \times O(1))) .
\end{aligned}
$$

Noticing that $V_{m, 1}=S^{m-1}$ and $G_{m-1,1}=R P^{m-1}$, we know that $q_{2}$ induces an isomorphism $q_{2 *} \pi_{m-1}\left(V_{m, 1}\right) \approx Z \longrightarrow \pi_{m-1}\left(G_{m-1,1}\right) \approx Z$ between the two systems of local coefficients. As is well-known, the coefficients in the first cohomology is the twisted integer $\widetilde{Z}$ when $q=m-1$, so is the coefficients in the second one. Thus $q_{2}$ induces an isomorphism

$$
q_{2 *}: H^{m}(B O(m) ; \widetilde{Z}) \longrightarrow H^{m}(B O(m) ; \widetilde{Z}) .
$$

Let $s$ be any $(m-1)$-skeleton section for the fibration $q_{1} q_{2}: B O(m-1) \longrightarrow$ $B O(m)$, then $q_{2} s$ is $(m-1)$-skeleton section for the fibration $q_{1}: B(O(m-$ 1) $\times O(1)) \longrightarrow B O(m)$. From Corollary 2.2.5, we obtain that

$$
q_{2 *}\left(\mathrm{ob}_{m-1}(s)\right)=\mathrm{ob}_{m-1}\left(q_{2} \circ s\right) .
$$

From [15], $\mathrm{ob}_{m-1}(s)$ is the primary obstruction for the vector bundle decomposition $\eta_{m} \approx \xi^{m-1} \oplus R$. From the primary obstruction theorem, the primary obstruction is independent with the choice of sections. Hence $\mathrm{ob}_{m-1}\left(q_{2} \circ s\right)$ is one of the primary obstruction for the decomposition $\eta_{m} \approx$ 
$\xi^{m-1} \oplus \lambda$. But $q_{2} *$ is an isomorphism, so we may regard $\mathrm{ob}_{m-1}(s)=$ $\mathrm{ob}_{m-1}\left(q_{2} \circ s\right) \in H^{m}(B O(m) ; \widetilde{Z})$.

In the following, we will prove that the other obstruction for the decomposition $\eta_{m} \approx \xi^{m-1} \oplus \lambda$ is either in $H^{m}(B O(m) ; \widetilde{Z})$ if $m$ is even, or in $H^{m}(B O(m) ; Z)$ if $m$ is odd. As in the proof of Theorem 2.1.11, there is an exact sequence:

$$
0 \longrightarrow \pi_{1}\left(G_{m-1,1}\right) \longrightarrow \pi_{1}(B(O(m-1) \times O(1))) \stackrel{q_{1 *}}{\longrightarrow} \pi_{1}(B(O(m))) \longrightarrow 0
$$

and $\pi_{1}\left(G_{m-1,1}\right) \approx \pi_{1}(B(O(m))) \approx Z_{2}$. Therefore the above sequence splits and there are exactly two splitting homomorphisms:

$$
\begin{aligned}
\theta_{1}, \theta_{2}: \pi_{1}(B O(m)) & \\
\quad \longrightarrow \pi_{1}(B(O(m-1) \times O(1))) & \approx \pi_{1}(B(O(m))) \oplus \pi_{1}\left(G_{m-1,1}\right)
\end{aligned}
$$

which are given by $\theta_{1}(\alpha)=(\alpha, 0)$ and $\theta_{2}(\alpha)=(\alpha, \beta)$, where $\alpha, \beta$ are the generators of $\pi_{1}(B O(m))$ and $\pi_{1}\left(G_{m-1,1}\right)$ respectively. We have already proved that $\theta_{1}(\alpha)=(\alpha, 0)$ corresponds to the obstruction class of the decomposition $\eta_{m} \approx \xi^{m-1} \oplus R$. In order to see the local coefficients determined by $\theta_{2}(\alpha)=(\alpha, \beta)$, we first recall the action of $\pi_{1}\left(G_{m-1,1}\right)$ on $\pi_{m-1}\left(G_{m-1,1}\right)$. The universal covering space of $G_{m-1,1}=R P^{m-1}$ is $S^{m-1}$. The only nontrivial covering translation is the antipodal map $h$ which corresponds to $\beta$. From [15], there is a commutative diagram

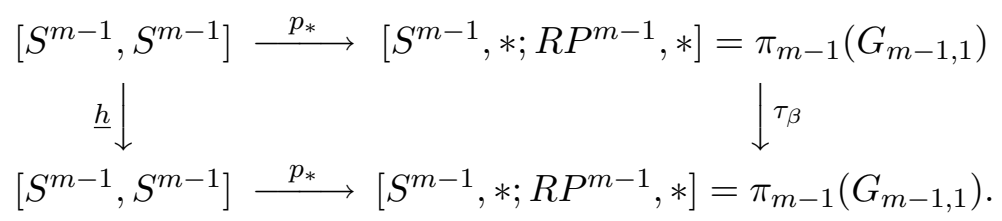

It is well-known that the degree of the antipodal map on $S^{m-1}$ is $(-1)^{m}$, from the above diagram, we know that the action of $\beta$ is given by

$$
\tau_{\beta}(\xi)=(-1)^{m} \xi \text {, for any } \xi \text { in } \pi_{m-1}\left(G_{m-1,1}\right)=Z \text {. }
$$

But the action of $(0, \beta)$ on $\pi_{m-1}\left(G_{m-1,1}\right)$ is the same as that of $\beta$, and the action of $(\alpha, 0)$ on $\pi_{m-1}\left(G_{m-1,1}\right)$ is the same as that of $\alpha$ which is the twisted action, i.e.,

$$
\tau_{\alpha}(\xi)=-\xi, \text { for any } \xi \text { in } \pi_{m-1}\left(G_{m-1,1}\right)=Z \text {. }
$$

Now the action induced by $\theta_{2}(\alpha)=(\alpha, \beta)$ is simply the product of the actions of $\alpha$ and $\beta$. So we have $\theta_{2}(\alpha)(\xi)=(-1)^{m+1} \xi$, and thus the action is either the twisted integr $\widetilde{Z}$ if $m$ is even or the ordinary integer $Z$ if $m$ is odd.

The most interesting part in the theorem is the following corollary: 
Corollary 2.2.7. There is an element $o_{2 n+1}$ in $H^{2 n+1}(B O(2 n+1) ; Z)$ such that for any map $f: X^{2 n+1} \longrightarrow B O(2 n+1)$, if $f^{*}\left(o_{2 n+1}\right)=0$, then $f^{*}\left(\eta_{2 n+1}\right) \approx \xi^{2 n} \oplus \lambda$, where $X^{2 n+1}$ is a $(2 n+1)$-dimensional CW-complex and $\eta_{2 n+1}$ is the universal vector bundle over $B O(2 n+1)$.

Proof. Let $o_{2 n+1}$ be the obstruction corresponding to the decomposition $\xi^{2 n} \oplus \lambda$ as in Theorem 2.2.6. By the primary obstruction theorem, there is a $(2 n)$-skeleton section $s$ for the fibration $p: B(O(2 n) \times O(1)) \rightarrow B O(2 n+$ 1) such that $o_{2 n+1}=\mathrm{ob}(s)$. By cellular approximation theorem, we may assume that $f$ is a cellular map. By the naturality of the primary obstruction class, we have

$$
f^{*}\left(o_{2 n+1}\right)=f^{*}(\mathrm{ob}(s))=\mathrm{ob}\left(f^{*} s\right)
$$

where $f^{*}(s)$ is the induced section by $f$ over the $(2 n)$-skeleton of $X^{2 n+1}$. So if $f^{*}\left(o_{2 n+1}\right)=0$, then $f^{*}(s)$ can be extended to a section over $X^{2 n+1}$, hence $f: X^{2 n+1} \longrightarrow B O(2 n+1)$ has a lifting for the fibration $p: B(O(2 n) \times$ $O(1)) \longrightarrow B O(2 n+1)$. By Lemma 2.1.1, $f^{*}\left(\eta_{2 n+1}\right) \approx \xi^{2 n} \oplus \lambda$.

The following corollary gives us some relationship between the decomposition obstruction and the well-known characteristic classes such as StiefelWhitney classes, Euler classes and Chern classes.

Corollary 2.2.8. If the coefficient is reduced to $Z_{2}$, then one of the two obstruction classes in Theorem 2.2.6 will be the top Stiefel-Whitney class of the universal bundle.

Proof. By definition, the top universal Stiefel-Whitney class can be defined as the primary obstruction class for the vector bundle decomposition $\eta_{m} \approx \xi^{m-1} \oplus R$ reduced the coefficient to $Z_{2}$. The corollary follows by Theorem 2.2.6.

Recall that the classifying space for oriented $m$-dimensional vector bundles is $B S O(m)$, and the inclusion $S O(m) \longrightarrow O(m)$ induced a universal covering map

$$
\pi: B S O(m) \longrightarrow B O(m)
$$

which is the classifying map for the universal oriented $m$-dimensional vector bundle $\zeta_{m}$ over $B S O(m)$.

Corollary 2.2.9. Let $\mathrm{OB}_{m-1, \theta_{1}}^{1}\left(\eta_{m}\right)$ and $\mathrm{OB}_{m-1, \theta_{2}}^{1}\left(\eta_{m}\right)$ be the two decomposition obstruction classes as in Theorem 2.2.6, and $\pi: B S O(m) \longrightarrow$ $B O(m)$ be the projection, then

$$
\pi^{*}\left(\mathrm{OB}_{m-1, \theta_{1}}^{1}\left(\eta_{m}\right)\right)=\pi^{*}\left(\mathrm{OB}_{m-1, \theta_{2}}^{1}\left(\eta_{m}\right)\right) \in H^{m}(B S O(m), Z),
$$

which is the Euler class of the universal oriented m-dimensional vector bundle $\zeta_{m}$ over $B S O(m)$. 
Proof. By the naturality of the obstruction classes, both $\pi^{*}\left(\mathrm{OB}_{m-1, \theta_{1}}^{1}\left(\eta_{m}\right)\right)$ and $\pi^{*}\left(\mathrm{OB}_{m-1, \theta_{2}}^{1}\left(\eta_{m}\right)\right)$ are the primary obstruction classes corresponding to the splitting homomorphism $\theta_{1} \pi_{*}$ and $\theta_{2} \pi_{*}$ for the codimension one decomposition of $\zeta_{m}$. But $B S O(m)$ is 1-connected, hence $\theta_{1} \pi_{*}=\theta_{2} \pi_{*}=0$. By the uniqueness of the primary obstruction class, we have $\pi^{*}\left(\mathrm{OB}_{m-1, \theta_{1}}^{1}\left(\eta_{m}\right)\right)=$ $\pi^{*}\left(\mathrm{OB}_{m-1, \theta_{2}}^{1}\left(\eta_{m}\right)\right) \in H^{m}(B S O(m), Z)$. Noticing that any line bundle over $B S O(m)$ is trivial, we see that $\pi^{*}\left(\mathrm{OB}_{m-1, \theta_{1}}^{1}\left(\eta_{m}\right)\right)$ is the obstruction for the decomposition $\zeta_{m}=\zeta^{m-1} \oplus R$. But Euler class can be defined to be the primary obstruction of the decomposition $\zeta_{m}=\zeta^{m-1} \oplus R$.

Recall that any $n$-dimensional complex vector bundle $\zeta^{n}$ can be regarded as a $2 n$-dimensional real vector bundle $\operatorname{Re}\left(\zeta^{n}\right)$. In terms of the structure groups, one has an inclusion $U(n) \subset O(2 n)$ which induces a fibration $p$ : $B U(n) \longrightarrow B O(2 n)$. If $\gamma_{n}$ is the universal $n$-dimensional complex vector bundle over $B U(n)$, then $\operatorname{Re}\left(\gamma_{n}\right)=p^{*}\left(\eta_{2 n}\right)$. Let $c_{n}\left(\zeta^{n}\right)$ be the $n$-th Chern class of a complex vector bundle $\zeta^{n}$, then we have the following corollary:

Corollary 2.2.10. With the above notations,

$$
\begin{aligned}
c_{m}\left(\gamma_{m}\right) & =p^{*}\left(\mathrm{OB}_{2 m-1, \theta_{1}}^{1}\left(\eta_{2 m}\right)\right) \\
& =p^{*}\left(\mathrm{OB}_{2 m-1, \theta_{2}}^{1}\left(\eta_{2 m}\right)\right) \in H^{2 m}(B U(m), Z) .
\end{aligned}
$$

Proof. Since $B U(m)$ is 1-connected, $\operatorname{Re}\left(\gamma_{n}\right)=p^{*}\left(\eta_{2 n}\right)$ is oriented vector bundle, as in Corollary 2.2.7, we know that

$$
p^{*}\left(\mathrm{OB}_{2 m-1, \theta_{1}}^{1}\left(\eta_{2 m}\right)\right)=p^{*}\left(\mathrm{OB}_{2 m-1, \theta_{2}}^{1}\left(\eta_{2 m}\right)\right),
$$

which is the Euler class of $\operatorname{Re}\left(\gamma_{n}\right)$. But the top Chern class of $\gamma_{n}$ is just the Euler class of $\operatorname{Re}\left(\gamma_{n}\right)$ (see $\left.[\mathbf{1 0}]\right)$.

In the following, we further consider the codimension 1 decomposition, that is the decomposition $\xi^{m} \approx \xi^{m-1} \oplus \lambda$. We try to begin from CWstructure of the classify space to see the restriction of the universal bundle to each cell.

Recall that a partition of $r \geq 0$ is an unordered sequence $\left(i_{1}, i_{2}, \ldots, i_{s}\right)$ of positive numbers such that the sum of the numbers is equal to $r$. For our purpose, we define a partition of $r \geq 0$ with length $n$ is an unordered sequence $\left(i_{1}, i_{2}, \ldots, i_{n}\right)$ of non-negative numbers such that the sum of the numbers is equal to $r$. We can always assume that $0 \leq i_{1} \leq i_{2} \leq \cdots \leq i_{n}$. Then there is an one to one correspondence $\left(i_{1}, i_{2}, \ldots, i_{n}\right) \leftrightarrow\left(\sigma_{1}, \sigma_{2}, \ldots, \sigma_{n}\right)$ given by $\sigma_{j}=i_{j}+j$, for $j=1,2, \ldots, n$. From [10], the Schubert symbol $\sigma=\left(\sigma_{1}, \sigma_{2}, \ldots, \sigma_{n}\right)$ determines an unique $r$-cell $e(\sigma)$, which is the set of $n$-planes in $R^{m}$ such that:

$$
e(\sigma)=\left\{X \mid \operatorname{dim}\left(X \cap R^{\sigma_{i}}\right)=i, \operatorname{dim}\left(X \cap R^{\sigma_{i-1}}\right)=i-1 ; i=1,2, \ldots, n\right\} .
$$


From [10], we know that the Grassmann manifold $G_{n}\left(R^{m}\right)$ has the following CW-structure:

$$
G_{n}\left(R^{m}\right)=\left\{\cup e(\sigma) \mid \sigma=\left(\sigma_{1}, \sigma_{2}, \ldots, \sigma_{n}\right)\right.
$$

is such that $\left.0<\sigma_{1}<\sigma_{2}<\cdots<\sigma_{n} \leq m\right\}$.

Taking the direct limit as $m \longrightarrow \infty$, one gets the infinite Grassmann manifold $G_{n}\left(R^{\infty}\right)$, which is the classifying space $B O(n)$.

Proposition 2.2.11. Let $e(\sigma)$ be a $(2 k+1)$-dimensional cell in $G_{n}\left(R^{\infty}\right)$, then

$$
\eta_{2 k+1} \mid \bar{e}(\sigma) \approx \xi^{2 k} \oplus \lambda
$$

where $\eta_{2 k+1}$ is the universal vector bundle over $G_{n}\left(R^{\infty}\right)$.

Proof. Let $\sigma_{j}=i_{j}+j$, for $j=1,2, \ldots, 2 k+1$, then

$$
\operatorname{dim} e(\sigma)=\sum_{j=1}^{2 k+1} i_{j}=2 k+1, \quad \text { and } \quad 0 \leq i_{1} \leq i_{2} \leq \ldots i_{2 k+1} .
$$

It is easy to see that the only $(2 k+1)$-cell such that $i_{1}>0$ is the cell with partition $(1,1, \ldots, 1)$. The corresponding Schubert symbol is $\sigma=$ $(2,3, \ldots, 2 k+2)$. By definition, $e(\sigma)$ consists of all the $(2 k+1)$-subspace $X$ in $R^{2 k+2}$ such that $\operatorname{dim}\left(X \cap R^{1}\right)=0$. It is not difficult to count all the faces of this cell, in fact, all the faces in the partition form are $(0,0, \ldots, 0,1, \ldots, 1)$. So the closure $\bar{e}(\sigma)$ for this cell is just $G_{2 k+1}\left(R^{2 k+2}\right)=R P^{2 k+1}$. And $\eta_{2 k+1} \mid G_{2 k+1}\left(R^{2 k+2}\right)$ is the canonical $(2 k+1)$-plane bundle of $G_{2 k+1}\left(R^{2 k+2}\right)$ which is the tangent bundle for $k>0$. It is well-known that there exists an nowhere 0 vector field for $\tau\left(S^{2 k+1}\right)$, in fact,

$$
\begin{aligned}
&\left(x_{0}, x_{1}, \ldots, x_{2 k}, x_{2 k+1}\right) \longrightarrow \\
&\left(\left(x_{0}, x_{1}, \ldots, x_{2 k}, x_{2 k+1}\right),\left(x_{1},-x_{0}, \ldots, x_{2 k+1},-x_{2 k}\right)\right)
\end{aligned}
$$

is such a vector field. It is easy to see that this vector field induces a section for the fibration $R P\left(\eta_{2 k+1} \mid \bar{e}(\sigma)\right) \longrightarrow \bar{e}(\sigma)$, the projective space bundle. By Lemma 2.1.3

$$
\eta_{2 k+1} \mid \bar{e}(\sigma) \approx \xi^{2 k} \oplus \lambda
$$

where $\sigma=(2,3, \ldots, 2 k+2)$.

For any other $(2 k+1)$-cell $e(\sigma), \sigma_{1}$ must be equal to 1 and each face of $e(\sigma)$ must also have the first entry 1 which means that $X \cap R^{1}=R^{1}$ for any $X \in \bar{e}(\sigma)$. So $R P\left(\eta_{2 k+1} \mid \bar{e}(\sigma)\right) \longrightarrow \bar{e}(\sigma)$ has a section given by $X \longrightarrow\left(X, R^{1}\right)$. Again by Lemma 2.1.3,

$$
\eta_{2 k+1} \mid \bar{e}(\sigma) \approx \xi^{2 k} \oplus \lambda
$$

where $\lambda$ can be even chosen to be trivial line bundle. Thus we complete the proof of the proposition. 


\section{Two examples.}

In this section, we will give two examples. In the first example, we demonstrate a method to calculate the obstruction classes. In the second one, we try to find as many as possible the trivial lines in the tangent bundle of a manifold up to cobordism.

Example 3.1. $H^{2 n}\left(R P^{2 n}, \widetilde{Z}\right) \approx Z$ and the generator is the obstruction class for the tangent bundle decomposition $\tau\left(R P^{2 n}\right) \approx \xi^{2 n-1} \oplus \lambda$.

Let $p: S^{2 n} \longrightarrow R P^{2 n}$ be the covering map and $\tau: S^{2 n} \longrightarrow S^{2 n}$ be the antipodal map. By Eilenberg Theorem $H^{2 n}\left(R P^{2 n}, \widetilde{Z}\right) \approx E^{2 n}\left(S^{2 n}, \widetilde{Z}\right)$, where $E^{2 n}\left(S^{2 n}, \widetilde{Z}\right)$ is the equivariant cohomology group which can be determined by the complex

$$
\left\{H_{p}\left(S^{p}, S^{p-1} ; Z\right) \stackrel{\partial}{\longrightarrow} H_{p-1}\left(S^{p-1}, S^{p-2} ; Z\right) \mid p=1,2, \ldots, 2 n\right\} .
$$

From [15], one can choose the orientation $e^{p}$ of the cell $E_{+}^{p}$, the upper hemisphere so that $H_{p}\left(S^{p}, S^{p-1} ; Z\right)=\left\langle e^{p}, \tau e^{p}\right\rangle$ and the boundary operator is given by

$$
\partial\left(e^{p}\right)=e^{p-1}+(-1)^{p} \tau e^{p-1}
$$

where $\tau e^{p}$ is the induced orientation on the cell of lower hemisphere by the antipodal map. As an equivariant group $H_{p}\left(S^{p}, S^{p-1} ; Z\right)$ has one generator $e^{p}$, so the set of equivariant homomorphism $\operatorname{Hom}^{Z_{2}}\left(H_{p}\left(S^{p}, S^{p-1} ; Z\right), \widetilde{Z}\right)$ has one generator $c_{e^{p}}$ which maps $e^{p}$ to 1 , where the antipodal map generates $Z_{2}$ and acts on integers by multliplying $(-1)$. Now we can calculate the coboundary operator:

$$
\delta\left(c_{e^{p}}\right)\left(e^{p+1}\right)=c_{e^{p}}\left(\partial e^{p+1}\right)=c_{e^{p}}\left(e^{p}+(-1)^{p+1} \tau e^{p}\right)=1+(-1)^{p}
$$

where we use the fact that $c_{e^{p}}\left(\tau e^{p}\right)=\tau\left(c_{e^{p}}\left(e^{p}\right)\right)=-1$. Now it is easy to see that $E^{2 n}\left(S^{2 n}, \widetilde{Z}\right)=Z$ with generator $c_{e^{p}}$. So $H^{2 n}\left(R P^{2 n}, \widetilde{Z}\right) \approx Z$.

In the following obstruction class for the decomposition $\tau\left(R P^{2 n}\right) \approx \xi^{2 n-1} \lambda$ is generator of $H^{2 n}\left(R P^{2 n}, \widetilde{Z}\right) \approx Z$. Consider the fibration

$$
p: R P\left(\tau\left(R P^{2 n}\right)\right) \longrightarrow R P^{2 n}
$$

whose fiber is $R P^{2 n-1}$. The obstruction for the existence of a section for $p$ is the same as that of for the decomposition $\tau\left(R P^{2 n}\right) \approx \xi^{2 n-1} \oplus \lambda$. If we have a section $s_{2 n-1}$ for $p: R P\left(\tau\left(R P^{2 n}\right)\right) \longrightarrow R P^{2 n}$ on the $(2 n-1)$-skeleton of $R P^{2 n}$. Let

$$
h:\left(B^{2 n}, S^{2 n-1}\right) \longrightarrow\left(R P^{2 n}, R P^{2 n-1}\right)
$$

be the characteristic map of the only $2 n$-cell of $R P^{2 n}$. In the pull-back diagram 




$h^{*}\left(R P\left(\tau\left(R P^{2 n}\right)\right)\right) \approx B^{2 n} \times R P^{2 n-1}$, since $B^{2 n}$ is contractible. The section $s_{2 n-1}$ induces a section for the trivial fibration $S^{2 n-1} \times R P^{2 n-1} \longrightarrow S^{2 n-1}$ which determines a map

$$
h^{\prime}: S^{2 n-1} \longrightarrow R P^{2 n-1} .
$$

The obstruction cocycle ob $\left(s_{2 n-1}\right)$ is just defined to be the correspondence:

$$
\mathrm{ob}\left(s_{2 n-1}\left(e^{2 n}\right)\right)=\left[h^{\prime}\right] \in \pi_{2 n-1}\left(R P^{2 n-1}\right) \approx Z
$$

which in turn determines an element

$$
\left[\mathrm{ob}\left(s_{2 n-1}\right)\right] \text { in } H^{2 n}\left(R P^{2 n}, \widetilde{\pi}_{2 n-1}\left(R P^{2 n-1}\right)\right) .
$$

We claim that the local coefficients $\widetilde{\pi}_{2 n-1}\left(R P^{2 n-1}\right)$ is the twisted integer $\widetilde{Z}$. To see this, we consider the natural inclusion:

$$
i: R P^{2 n}=G_{2 n}\left(R^{2 n+1}\right) \subset G_{2 n}\left(R^{\infty}\right)=B O(2 n) .
$$

From Theorem 2.2.6, the obstruction for the decomposition $\eta_{2 n} \approx \xi^{2 n-1} \oplus \lambda$ is in

$$
H^{2 n}(B O(2 n) ; \widetilde{Z})
$$

But the inclusion $i$ induces an isomorphism on the fundamental groups:

$$
i_{*}: \pi_{1}\left(G_{2 n}\left(R^{2 n+1}\right)\right) \approx \pi_{1}\left(G_{2 n}\left(R^{\infty}\right)\right) \approx Z_{2} .
$$

By the naturality of the decomposition obstruction classes, $i$ induces a homomorphism:

$$
i^{*}: H^{2 n}\left(G_{2 n}\left(R^{\infty}\right), \widetilde{Z}\right) \longrightarrow H^{2 n}\left(G_{2 n}\left(R^{2 n+1}\right), i_{*} \widetilde{Z}\right)
$$

which maps the decomposition obstructions of $\eta_{2 n}$ to that of $i *\left(\eta_{2 n}\right)=$ $\tau\left(R P^{2 n}\right)$. So $\widetilde{\pi}_{2 n-1}\left(R P^{2 n-1}\right) \approx i_{*} \widetilde{Z}=\widetilde{Z}$. Thus

$$
\left[\mathrm{ob}\left(s_{2 n-1}\right)\right] \in H^{2 n}\left(R P^{2 n}, \widetilde{\pi}_{2 n-1}\left(R P^{2 n-1}\right)\right) \approx H^{2 n}\left(R P^{2 n}, \widetilde{Z}\right) .
$$

In order to calculate $\left[h^{\prime}\right] \in \pi_{2 n-1}\left(R P^{2 n-1}\right) \approx Z$, we need to choose a specific characteristic map $h$ for the $2 n$-cell, and find the trivilization of the pull-back of fibration $p: R P\left(\tau\left(R P^{2 n}\right)\right) \longrightarrow R P^{2 n}$. Let

$$
q: V_{k}\left(R^{m}\right) \longrightarrow G_{k}\left(R^{m}\right)
$$

be the principal $O(k)$-bundle, where $V_{k}\left(R^{m}\right)$ is the Stiefel manifold, then the pull-back of the canonical $k$-bundle over $G_{k}\left(R^{m}\right)$ by $q$ is isomorphic to the trivial bundle

$$
V_{k}\left(R^{m}\right) \times R^{k} \longrightarrow V_{k}\left(R^{m}\right) .
$$


The isomorphism is given by

$$
\left(\left(\nu_{1}, \nu_{2}, \ldots, \nu_{k}\right),\left(t_{1}, t_{2}, \ldots, t_{k}\right)\right) \longrightarrow\left(\left(\nu_{1}, \nu_{2}, \ldots, \nu_{k}\right),\left(\langle V\rangle, \sum_{1}^{k} t_{i} \nu_{i}\right)\right)
$$

where $\nu_{1}, \nu_{2}, \ldots, \nu_{k}$ are $k$ unit orthogonal vectors in $R^{m}$, and $\langle V\rangle$ is the $k$ dimensional subspace in $R^{m}$ generated by $\nu_{1}, \nu_{2}, \ldots, \nu_{k}$. The inverse is given by

$$
\left(\left(\nu_{1}, \nu_{2}, \ldots, \nu_{k}\right),(\langle V\rangle, \nu)\right) \longrightarrow\left(\left(\nu_{1}, \nu_{2}, \ldots, \nu_{k}\right),\left(\nu \cdot \nu_{1}, \nu \cdot \nu_{2}, \ldots, \nu \cdot \nu_{k}\right)\right) .
$$

So any vector bundle factors out though $q: V_{k}\left(R^{m}\right) \longrightarrow G_{k}\left(R^{m}\right)$ is trivial and the trivilization is given by the above isomorphisms. In particular, we know the trivilization on any subspace of $V_{k}\left(R^{m}\right)$. The following theorem [10] states that the characteristic map for any cell in $G_{k}\left(R^{m}\right)$ can be chosen to be the restriction of $q: V_{k}\left(R^{m}\right) \longrightarrow G_{k}\left(R^{m}\right)$ to some subspace of $V_{k}\left(R^{m}\right)$.

Theorem 3.2. In the $\mathrm{CW}$-structure of $G_{n}\left(R^{m}\right)$,

$$
\begin{aligned}
& G_{n}\left(R^{m}\right)=\left\{\cup e(\sigma) \mid \sigma=\left(\sigma_{1}, \sigma_{2}, \ldots, \sigma_{n}\right)\right. \\
& \left.\quad \text { is such that } 0<\sigma_{1}<\sigma_{2} \cdots<\sigma_{n} \leq m\right\}
\end{aligned}
$$

the characteristic map of the cell $e(\sigma)$ can be given by

$$
\left.q\right|_{\bar{H}^{\sigma_{1}} \times \cdots \times \bar{H}^{\sigma_{n}}}: V_{n}\left(R^{m}\right) \cap \bar{H}^{\sigma_{1}} \times \cdots \times \bar{H}^{\sigma_{n}} \longrightarrow G_{n}\left(R^{m}\right)
$$

where $\bar{H}^{\sigma_{1}}=\left\{\left(x_{1}, x_{2}, \ldots, x_{\sigma_{i}}, 0, \ldots, 0\right) \in R^{m} \mid x_{\sigma_{i}} \geq 0\right\}$.

Before further considering the characteristic map for the top cell in $G_{2 n}\left(R^{2 n+1}\right)$, we need the following lemma:

Lemma 3.3. Let $\left(\nu_{1}, \nu_{2}, \ldots, \nu_{k}\right) \in V_{k}\left(R^{k+1}\right)$ be written in matrix form:

$$
\left[\begin{array}{c}
\nu_{1} \\
\nu_{2} \\
\vdots \\
\nu_{k}
\end{array}\right]=\left[\begin{array}{cccc}
\nu_{1,1} & \nu_{1,2} & \cdots & \nu_{1, k+1} \\
\nu_{2,1} & \nu_{2,2} & \cdots & \nu_{2, k+1} \\
\cdots & \cdots & \cdots & \cdots \\
\nu_{k, 1} & \nu_{k, 2} & \cdots & \nu_{k, k+1}
\end{array}\right]
$$

then the vector $\left(\nu_{1}, \ldots, \nu_{k}\right)^{\perp}=\left(A_{1}, A_{2}, A_{k+1}\right)$, where

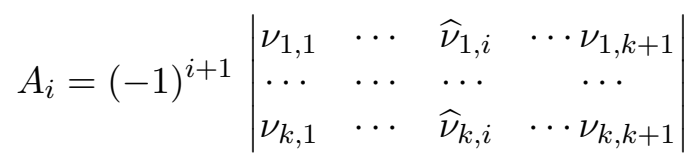

is an unit vector and orthogonal to each $\nu_{i}$ for $1,2, \ldots, k$. 
Proof. Let $w=\left(w_{1}, w_{2}, \ldots, w_{k+1}\right)$ be the unique unit vector that is orthogonal to each $\nu_{i}$ for $i=1,2, \ldots, k$ and such that

$$
\left|\begin{array}{ccccc}
\nu_{1,1} & \cdots & \nu_{1, i} & \cdots & \nu_{1, k+1} \\
\cdots & \cdots & \cdots & \cdots & \cdots \\
\nu_{k, 1} & \cdots & \nu_{k, i} & \cdots & \nu_{k, k+1} \\
w_{1} & \cdots & w_{k, i} & \cdots & w_{k+1}
\end{array}\right|=(-1)^{k} .
$$

From the determinant properties we know that $\left(\nu_{1}, \ldots, \nu_{k}\right)^{\perp}=\left(A_{1}, A_{2}, \ldots\right.$, $\left.A_{k+1}\right)$ is orthoganal to each $\nu_{i}$ for $i=1,2, \ldots, k$ and so

$$
\left(\nu_{1}, \ldots, \nu_{k}\right)^{\perp}=t w
$$

hence $\left\langle\left(\nu_{1}, \ldots, \nu_{k}\right)^{\perp}, w\right\rangle=t\langle w, w\rangle=t$. Expanding the determinant $(*)$ on the last row, we see that $(-1)^{k} \sum_{i=1}^{k+1} w_{i} A_{i}=(-1)^{k}$, that is $\left\langle\left(\nu_{1}, \ldots, \nu_{k}\right)^{\perp}, w\right\rangle$ $=1$. Thus we proved that $\left(\nu_{1}, \ldots, \nu_{k}\right)^{\perp}=w$ and hence $\left(\nu_{1}, \ldots, \nu_{k}\right)^{\perp}$ is an unit vector and orthogonal to each $\nu_{i}$ for $i=1,2, \ldots, k$.

Now we consider the $2 n$-cell in $G_{2 n}\left(R^{2 n+1}\right)$ which corresponds to the Schubert symbol $(2,3, \ldots, 2 n+1)$. In this special case, we can further give a specific homeomorphism:

$$
h_{2 n}: I^{2 n} \longrightarrow V_{2 n}\left(R^{2 n+1}\right) \cap \bar{H}^{2} \times \cdots \times \bar{H}^{2 n+1}
$$

by

$$
h_{2 n}\left(x_{1}, x_{2}, \ldots, x_{2 n}\right)=\left(\nu_{1}, \nu_{2} \ldots, \nu_{2 n}\right)
$$

where

$$
\begin{aligned}
& \nu_{1}=x_{1} e_{1}+\sqrt{1-x_{1}^{2}} e_{2} \\
& \nu_{2}=x_{2}\left(\sqrt{1-x_{1}^{2}} e_{1}-x_{1} e_{2}\right)+\sqrt{1-x_{2}^{2}} e_{3} \\
& \ldots \ldots \ldots \ldots \\
& \nu_{k}=x_{k}\left(\nu_{1}, \ldots \nu_{k-1}\right)^{\perp}+\sqrt{1-x_{k}^{2}} e_{k+1}
\end{aligned}
$$

with inverse given by

$$
h_{2 n}^{-1}\left(\nu_{1}, \nu_{2}, \ldots, \nu_{2 n}\right)=\left(\left\langle\nu_{1}, e_{1}\right\rangle,\left\langle\nu_{2},\left(\nu_{1}\right)^{\perp}\right\rangle, \ldots,\left\langle\nu_{2 n},\left(\nu_{1}, \ldots, \nu_{2 n-1}\right)^{\perp}\right\rangle\right) .
$$

Now we consider the $(2 n-1)$-skeleton section $s_{2 n-1}$ for the fibration

$$
p: R P\left(\tau\left(R P^{2 n}\right)\right) \longrightarrow R P^{2 n}=G_{2 n}\left(R^{2 n+1}\right)
$$

given by

$$
s_{2 n-1}(X)=\left(X,\left[e_{1}\right]\right) .
$$

In fact, for any cell $e(\sigma)$ in $B O(2 n)$ and $X \in e(\sigma), \operatorname{dim}\left(X \cap R^{1}\right)=1$ if $\operatorname{dim} e(\sigma)<2 n$. So $s_{2 n-1}$ is a section over the $(2 n-1)$-skeleton for the fibration

$$
R P\left(\eta_{2 n}\right) \longrightarrow B O(2 n)
$$


Now we can calculate the obstruction cocycle for the section $s_{2 n-1}$ as follows: In the pull-back diagram

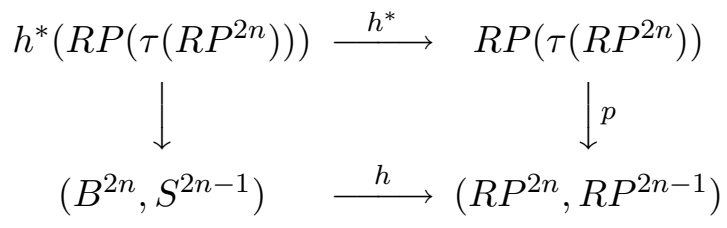

if we choose the characteristic map to be

$$
q h_{2 n}: I^{2 n} \longrightarrow V_{2 n}\left(R^{2 n+1}\right) \cap \bar{H}^{2 n+1} \times \cdots \times \bar{H}^{2 n+1} \longrightarrow G_{2 n}\left(R^{2 n+1}\right)
$$

then the pull-back diagram will be equivalent to the following diagram



where $I^{2 n} \times R P^{2 n-1} \stackrel{q h_{2 n}^{*}}{\longrightarrow} R P\left(\tau\left(R P^{2 n}\right)\right)$ is given by

$$
q h_{2 n}^{*}\left(\left(x_{1}, \ldots, x_{2 n}\right),\left[t_{1}, \ldots, x_{2 n}\right]\right)=\left(q h_{2 n}\left(x_{1}, \ldots, x_{2 n}\right), \sum_{i} t_{i} \nu_{i}\right)
$$

where $h_{2 n}\left(x_{1}, x_{2}, \ldots, x_{2 n}\right)=\left(\nu_{1}, \nu_{2}, \ldots, \nu_{2 n}\right)$. The induced section $s_{2 n-1}^{*}$ over $\partial\left(I^{2 n}\right) \approx S^{2 n-1}$ is given by

$$
s_{2 n-1}^{*}\left(x_{1}, \ldots, x_{2 n}\right)=\left(\left(x_{1}, \ldots, x_{2 n}\right),\left[\left\langle e_{1}, \nu_{1}\right\rangle, \ldots\left\langle e_{1}, \nu_{2 n}\right\rangle\right]\right)
$$

which induces a map $h: \partial\left(I^{2 n}\right) \approx S^{2 n-1} \longrightarrow R P^{2 n-1}$ given by

$$
h\left(x_{1}, \ldots, x_{2 n}\right)=\left[\left\langle e_{1}, \nu_{1}\right\rangle, \ldots,\left\langle e_{1}, \nu_{2 n}\right\rangle\right] .
$$

Taking a close look at the formula for $h_{2 n}\left(x_{1}, x_{2}, \ldots, x_{2 n}\right)=\left(\nu_{1}, \nu_{2}, \ldots, \nu_{2 n}\right)$, we find that

$$
\left[\left\langle e_{1}, \nu_{1}\right\rangle, \ldots,\left\langle e_{1}, \nu_{2 n}\right\rangle\right]=\left[x_{1}, x_{2} \sqrt{1-x_{1}^{2}}, \ldots, x_{2 n} \sqrt{1-x_{1}^{2}} \cdots \sqrt{1-x_{2 n-1}^{2}}\right] .
$$

Thus $h\left(x_{1}, \ldots, x_{2 n}\right)=\left[x_{1}, x_{2} \sqrt{1-x_{1}^{2}}, \ldots, x_{2 n} \sqrt{1-x_{1}^{2}} \cdots \sqrt{1-x_{2 n-1}^{2}}\right]$ which is homotopic to the map $\left(x_{1}, \ldots, x_{2 n}\right) \longrightarrow\left[x_{1}, \ldots, x_{2 n}\right]$ via the following homotopy

$$
H_{t}\left(x_{1}, \ldots, x_{2 n}\right)=\left[x_{1}, x_{2} \sqrt{1-t x_{1}^{2}}, \ldots, x_{2 n} \sqrt{1-t x_{1}^{2}} \cdots \sqrt{1-t x_{2 n-1}^{2}}\right] .
$$

Therefore $[h]$ represents the generator in $\pi_{2 n-1}\left(R P^{2 n-1}\right)$, hence the obstruction class $\left[\mathrm{ob}\left(s_{2 n-1}\right)\right]$ for the decomposition $\tau\left(R P^{2 n}\right) \approx \xi^{2 n-1} \oplus \lambda$ is the generator in $H^{2 n}\left(R P^{2 n} \widetilde{Z}\right) \approx Z$. Thus we complete our first example 
In the following example, we will see that the decomposition $\xi^{2 n+1} \approx$ $\xi^{2 n} \oplus \lambda$ is often possible. Let $M O_{*}$ denote the Thom cobordism ring. It is well-known that $M O_{*}=\sum_{n \geq 0} M O_{n}=Z_{2}\left[X_{n} \mid n \neq 2^{k}-1\right]$ is a graded polynomial algebra over $Z_{2}$ with one generator in each dimension $n$ not in the form $2^{k}-1$ for all $k>0$.

Example 3.4. Let $\left[M_{2 k+1}\right] \in M O_{2 k+1}$ be a $(2 k+1)$-dimensional cobordism class, then we can choose $M_{2 k+1}$ such that $\tau\left(M_{2 k+1}\right) \approx \xi^{2 k} \oplus R$.

Proof. For any odd dimensional generator, we will choose such a representative. Consider the vector bundle $\lambda_{1} \oplus \cdots \oplus \lambda_{m}$ over $R P\left(n_{1}\right) \times \cdots \times R P\left(n_{m}\right)$, where $\lambda_{1}$ is the pull-back of the canonical line bundle over $i$-th factor. Let $R P\left(n_{1}, \ldots, n_{m}\right)$ be the projective space bundle of $\lambda_{1} \oplus \cdots \oplus \lambda_{m}$, then it is a $n$-dimensional smooth manifold, where $n=\sum_{1}^{m} n_{i}+m-1$. From [13], $R P\left(n_{1}, \ldots, n_{m}\right)$ is indecomposable in $M O_{n}$ if and only if $\left(\begin{array}{c}n-1 \\ n_{1}\end{array}\right)+\cdots+$ $\left(\begin{array}{c}n-1 \\ n_{m}\end{array}\right)=1 \bmod 2$.

Let $n$ be a positive odd number that is not in the form $2^{k}-1$, then $n$ can be uniquely written as $n=2^{p+1} q+2^{p}-1$, where $p, q$ are positive integers. Let $X_{n}$ be the manifold $R P\left(2^{p}, 1, \ldots, 1,0\right)$ where the number of 1 's is $2^{p} q-1$ which is greater than 0 . Noticing that $2^{p}$ and 1 do not appear in the binary expression of $n-1$, one can check that $R P\left(2^{p}, 1, \ldots, 1,0\right)$ is indecomposable. From Borel-Hirzebruch [4], the tangent bundle of the projective space bundle $R P(\xi)$ associated with a vector bundle $\xi$ over a smooth manifold $M$ always splits:

$$
\tau(R P(\xi)) \approx p^{*}(\tau(M)) \oplus \tau_{2}
$$

where $p: R P(\xi) \longrightarrow M$ is the projection, and $\tau_{2}$ is the bundle along the fiber. From this result, noticing that the tangent bundle of $R P^{1}$ is trivial, we see that the tangent bundle of $R P\left(2^{p}, 1, \ldots, 1,0\right)$ has a $\left(2^{p} q-1\right)$-dimensional trivial summand.

For even number $n$, we may just choose $R P^{n}$ to be the generator. From the polynomial structure of $M O_{*}$, any $\left[M_{2 k+1}\right] \in M O_{2 k+1}$ has the form:

$$
\left[M_{2 k+1}\right]=\sum_{I} \varepsilon_{I} X_{i_{1}} \cdots X_{i_{r}}
$$

where $\varepsilon \in Z_{2}$, and $\sum_{j=1}^{r} i_{j}=2 k+1$. So at least one of the generator has odd dimension. Therefore the tangent bundle of $\sum_{I} \varepsilon_{I} X_{i_{1}} \cdots X_{i_{r}}$ has a trivial summand.

Since the characteristic numbers are cobordism invariants, noticing that $w_{2 n+1}\left(\xi^{2 n} \oplus R\right)=0$, and using the above example, we get a different proof of the fact that $\left\langle w_{2 n+1}\left[M^{2 n+1}\right]\right\rangle=0$ for any odd dimensional closed manifold $M^{2 n+1}$. 
Acknowledgement. The second author would like to thank Dr. Clement Lutterodt for his various kinds of helps. He also wishes to thank Dr. Sundararaman, Dr. Einstein-Matthews, Dr. Seydi and Dr. Banyaga for their encouragement.

\section{References}

[1] M. Atiyah and E. Rees, Vector bundles on projective 3-space, Invent. Math., 35 (1976), 131-151, MR 54 \#7870, Zbl 0332.32020.

[2] W. Barth and A. Van de Ven, A decomposition criterion for algebraic 2-bundle on projective space, Invent. Math., 25 (1974), 91-106, MR 52 \#420, Zbl 0295.14006.

[3] H.J. Baues, Obstruction Theory, Lecture Notes in Mathematics, 628, Springer, New York, 1977, MR 57 \#7600, Zbl 0361.55017.

[4] A. Borel and F. Hirzebruch, On the characteristic classes of homogeneous spaces I, II, Amer. J. Math., 80 (1958), 458-538; 81 (1959), 315-382, MR 21 \#1586, MR 22 \#98, Zbl 0097.36401.

[5] B. Eckmann and P.S. Hilton, Homotopical obstruction theory, An. Acad. Brasil Ci., 40 (1968), 407-425, MR 44 \#7546, Zbl 0174.26201.

[6] F. Hirzebruch, Topological Methods in Algebraic Geometry, Springer, Berlin, Heidelberg, New York, 1966, MR 34 \#2573, Zbl 0138.42001.

[7] S. Jackowski, J.E. McClure and B. Oliver, Self-maps of classifying spaces of compact simple Lie groups, Bull. Amer. Math. Soc., 22(1) (1990), 65-72, MR 90f:55023, Zbl 0692.55013.

[8] _ Maps between classifying spaces revisited, Contemporary Mathematics, 181 (1995), 263-298, MR 96a:55027, Zbl 0832.55013.

[9] J.F. McClendon, Obstruction theory in fiber spaces, Math. Z., 120 (1971), 1-17, MR 45 \#6002, Zbl 0222.55023.

[10] J.W. Milnor and J.D. Stasheff, Characteristic Classes, Ann. of Math. Studies, 76, Princeton University Press and University of Tokyo Press, 1974, MR 55 \#13428, Zbl 0298.57008.

[11] D. Notbohm, Maps between classifying spaces and applications, J. Pure Appl. Algebra, 3 (1993), 273-294, MR 95c:55019, Zbl 0794.55011.

[12] R.L.E. Schwarzenberger, Vector bundles over the projective plane, Proc. London Math. Soc. (3), 11 (1961), 623-640, MR 25 \#1161, Zbl 0212.26004.

[13] R. Stong, On fibering of cobordism classes, Trans. Amer. Math. Soc., 178 (1973), 431-447, MR 47 \#4282, Zbl 0267.57025.

[14] R. Switzer, Complex 2-plane bundle over complex projective space, Math. Z., 168 (1979), 275-287, MR 80j:55022, Zbl 0394.55019.

[15] G.W. Whitehead, Elements of Homotopy Theory, Springer-Verlag, New York, Heidelberg, Berlin, 1978, MR 80b:55001, Zbl 0406.55001

Received October 25, 1999. 
E-mail address: jleslie@howard.edu

Department of Mathematics

HOWARD UNIVERSITY

WASHington, DC 20059 